\title{
Pore-Size Measurement from Eigenvalues of Magnetic Resonance Relaxation
}

\author{
Afrough, Armin; Marica, Florea; Macmillan, Bryce; Balcom, Bruce J.
}

Published in:

Physical Review Applied

Link to article, DOI:

10.1103/PhysRevApplied.16.034040

Publication date:

2021

Document Version

Publisher's PDF, also known as Version of record

Link back to DTU Orbit

Citation (APA):

Afrough, A., Marica, F., Macmillan, B., \& Balcom, B. J. (2021). Pore-Size Measurement from Eigenvalues of Magnetic Resonance Relaxation. Physical Review Applied, 16(3), [034040].

https://doi.org/10.1103/PhysRevApplied.16.034040

\section{General rights}

Copyright and moral rights for the publications made accessible in the public portal are retained by the authors and/or other copyright owners and it is a condition of accessing publications that users recognise and abide by the legal requirements associated with these rights.

- Users may download and print one copy of any publication from the public portal for the purpose of private study or research.

- You may not further distribute the material or use it for any profit-making activity or commercial gain

- You may freely distribute the URL identifying the publication in the public portal

If you believe that this document breaches copyright please contact us providing details, and we will remove access to the work immediately and investigate your claim. 


\title{
Pore-Size Measurement from Eigenvalues of Magnetic Resonance Relaxation
}

\author{
Armin Afrough $\odot,{ }^{1,2}$ Florea Marica $\odot,{ }^{1}$ Bryce MacMillan $\odot,{ }^{1}$ and Bruce J. Balcom ${ }^{1, *}$ \\ ${ }^{1}$ UNB MRI Research Centre, Department of Physics, University of New Brunswick, Fredericton, New Brunswick, \\ Canada E3B $5 A 3$ \\ ${ }^{2}$ Danish Hydrocarbon Research and Technology Centre, Technical University of Denmark, Elektrovej, Kongens \\ Lyngby 2800, Denmark
}

(Received 10 February 2021; revised 16 July 2021; accepted 16 August 2021; published 22 September 2021)

\begin{abstract}
Nonground eigenvalues are widely disregarded in magnetic resonance relaxation measurements of porous media due to difficulties involved in their measurement, detection, and the derivation of physically meaningful parameters from them. Such nonground eigenvalues may be experimentally observed in relaxation measurements, such as the relaxation correlation of $T_{1}-T_{2}$, and yield information on the pore size and surface relaxivity of porous media without calibration through other independent measurements. Nonground eigenvalue analysis of $T_{1}-T_{2}$ measurements on Berea sandstone undertaken at three static magnetic fields produces pore sizes consistent with those obtained through x-ray microtomography and SEM measurements. Similar agreement is found for a Bentheimer sandstone with a more complex pore geometry. A phase-encoding imaging variant of this method measures the imbibition confinementsize profile in Berea sandstone. It is suggested that the existence of nonground eigenmodes may be much more prevalent in simple magnetic resonance relaxation measurements than previously considered. Therefore, it is possible to measure pore size by matching numerical Brownstein-Tarr solutions with those of experiments in a wide variety of samples and magnetic resonance methods.
\end{abstract}

DOI: 10.1103/PhysRevApplied.16.034040

\section{INTRODUCTION}

Magnetic resonance has become an indispensable technique in probing porous rocks, especially in well logging [1], petrophysics [2], and hydrogeology [3]. These methods acquire information on pores [4], pore-filling fluids [5], and related fluid dynamics [6]. Magnetic resonance methods may be integrated with routine industrial and research protocols on investigating samples of industry-standard size [7], 1.5 inch or $38 \mathrm{~mm}$ in diameter and $50-70 \mathrm{~mm}$ in length, to observe micrometer-scale features and phenomena. This is in contrast with the sample size reduction and sample-preparation requirements of x-ray microtomography and scanning electron microscopy, respectively. In addition to bulk measurements, a number of quantitative imaging methods are specially developed for laboratory studies of rocks (see reviews [2] and [8]) that may also be employed at elevated pressures and temperatures in dynamic experiments $[9,10]$.

Relaxation methods are the most common class of magnetic resonance measurements applied to rock samples. Surface water, water residing on the pore surface, has a short relaxation time due to several relaxation mechanisms, including homonuclear dipole-dipole coupling,

\footnotetext{
*bjb@unb.ca
}

cross-relaxation by other nuclear spins, relaxation by paramagnetic ions, and relaxation by free electrons [11]. Surface water has a relaxation time in the order of $1 \mathrm{~ms}$ [12] compared with bulk water, which has a relaxation time of approximately $3 \mathrm{~s}$ at room temperature. Zimmerman and Brittin [12] described this two-site exchange and predicted the observed relaxation rate for various exchange rates of very slow, very fast, and in between. It is usually assumed that exchange between surface water and bulk water in pores is fast [13]. The prevalent two-site fast-exchange model therefore leads to a linear relationship between pore size, $l$, and longitudinal, $T_{1}$, and transverse, $T_{2}$, relaxationtime constants for common rock samples in laboratory settings.

Inverse Fredholm integrals of the first kind transform time-domain relaxation decays into relaxation-time distributions that display signal population as a function of their relaxation time. These $T_{1}$ and $T_{2}$ distributions are usually regarded as proxies for the pore-size distribution, due to the perceived linear relationship between pore size and relaxation-time constants. The relaxation-time axis is, however, seldom transformed to a pore-size axis. It is common to observe two or three peaks in relaxationtime distributions of rocks, even when they have only one dominant pore size [14]. In such cases, there is always a dominant peak with shoulder peaks of shorter relaxation time and lower intensity. This work 
demonstrates that low-intensity peaks commonly observed in $T_{1}$ and $T_{2}$ distributions may be nonground eigenvalues of the diffusion-relaxation equation, as theoretically predicted by Brownstein and Tarr [15].

Brownstein and Tarr [15] demonstrated the effect of diffusion in magnetic resonance relaxation in porous media. They expand the solution of magnetization evolution in the diffusion-relaxation equation as a series of orthogonal spatial functions that decay exponentially with time. The solution of Zimmerman and Brittin approximates the first term in such a solution, the ground relaxation-time eigenvalue. Higher-order decay rates, corresponding to nonground eigenvalues, have shorter time constants and lower intensities. These nonground eigenvalues describe complex diffusion behavior in pores and contain information about geometrical features of the investigated medium, such as pore size.

In the quest for methods that reveal nonground eigenvalues of magnetic resonance in porous media, the group of Song concentrated on creating inhomogeneous magnetization in porous samples that predominantly excited nonground eigenvalues [4,16-19]. For this purpose, they exploited internal magnetic field gradients. Their measurement method did not gain wide currency due to its apparent complexity in implementation and analysis. Other notable attempts in this regard, by the groups of Davies [20,21], Keating [22-24], Müller-Petke [25-27], and Yu [28] recognized the contribution of nonground eigenvalues to magnetic resonance relaxation; however, they did not fully explore the applications of this method, especially in complex natural porous media.

Most investigators assume fast-diffusion behavior in magnetic resonance relaxation in porous materials. This assumption may be erroneous and nonground eigenvalues may actually contribute to magnetic resonance relaxation signals. Estimates of the pore size measured by employing such methods will be incorrect with an incorrect assumption. Brownstein and Tarr described three qualitative regions of behavior for eigenvalues of magnetic resonance relaxation according to dimensionless number $\rho l / D$, where $l$ is the pore size, $\rho$ is the surface relaxivity, and $D$ is the mass diffusivity. Fast-diffusion and intermediate-diffusion regimes, respectively, correspond to $\rho l / D \ll 1$ and $1 \ll \rho l / D \ll 10$. For typical sandstones, $l=40 \mu \mathrm{m}, \rho_{1}=25 \mu \mathrm{m} / \mathrm{s}, D=2.5 \times 10^{-9} \mathrm{~m}^{2} / \mathrm{s}$ (see Table 2 in Ref. [29]), and $\rho_{1} l / D=0.4$, which is close to the intermediate-diffusion regime. Since $\rho_{2}>\rho_{1}$, it is expected that transverse-relaxation-time distributions of rocks are either in, or very close to, the intermediatediffusion regime.

Independently, we recognized that nonground eigenvalues of the relaxation-diffusion equation in magnetic resonance relaxation of porous media might be experimentally observed in simple measurements that commenced with homogeneous magnetization [30]. Such measurements, including that of Carr-Purcell-Meiboom-Gill (CPMG), inversion recovery, and their variants, are extensively employed worldwide. Nonground eigenvalues of relaxation, after detection, may be employed in predicting pore size and surface relaxivities in natural porous media. This paper expands our previous letter [30] with more detailed descriptions, more experiments, different samples, three static magnetic fields, and a $T_{1}-T_{2}$ imaging method.

Nonground eigenvalues are widely disregarded in magnetic resonance relaxation of porous media, although Brownstein and Tarr introduced them four decades ago. This work provides a long-overdue interpretation of nonground eigenvalues in common magnetic resonance relaxation experiments in porous media. This work also provides a means to reprocess a vast amount of extant relaxation data and motivates the search for measurement methods that permit better measurement of nonground eigenvalues in complex multimodal systems.

\section{THEORY}

Torrey [31] generalized the Bloch equations [32] by the addition of terms that described magnetization transfer by diffusion. The general Bloch-Torrey equations simplify for a homogeneous magnetic field in the $z$ direction, so that longitudinal relaxation occurs parallel to the external magnetic field,

$$
\left(\frac{\partial}{\partial t}-D \nabla^{2}+\frac{1}{T_{1 b}}\right) M_{z}(\mathbf{r}, t)=\frac{M_{0}}{T_{1 b}},
$$

and transverse relaxation occurs perpendicular to the magnetic field,

$$
\left(\frac{\partial}{\partial t}-D \nabla^{2}+\frac{1}{T_{2 b}}\right) M_{+}(\mathbf{r}, t)=0
$$

In Eqs. (1) and (2), $M_{+}=M_{x}+i M_{y}, T_{1 b}$ and $T_{2 b}$ are bulk relaxation-time constants of the pore-filling fluid, $M_{0}$ is equilibrium magnetization, and $D$ is isotropic diffusivity. Appropriate initial and boundary conditions are necessary to solve magnetization evolution in pores.

Korringa et al. [33] modeled magnetic resonance relaxation enhancement of liquids restricted by solid surfaces with sparsely distributed electron spins. Although a proper description of relaxation dependence on concentration, temperature, and magnetic field may be obtained, such models do not yield simple boundary conditions. Brownstein and Tarr [15] demonstrated that knowing the relaxation capacity of the surface as longitudinal- and transverse-surface relaxivities, $\rho_{1}$ and $\rho_{2}$, was sufficient for writing boundary conditions

$$
\left(D \hat{\mathbf{n}} \cdot \nabla+\rho_{1}\right) M_{z}(\mathbf{r}, t)=0
$$


and

$$
\left(D \hat{\mathbf{n}} \cdot \nabla+\rho_{2}\right) M_{+}(\mathbf{r}, t)=0,
$$

for Eqs. (1) and (2), respectively. Surface relaxivities may be heterogeneous in complex porous media. They are, however, assumed to be homogeneous here, and $\rho_{1}$ and $\rho_{2}$ are treated as scalars.

The initial conditions for Eqs. (1) and (2) depend on the pulse sequence employed. Simple magnetic resonance relaxation experiments commence with a homogeneous magnetization of

$$
M_{z}(\mathbf{r}, t)=-M_{0},
$$

or

$$
M_{+}(\mathbf{r}, t)=M_{0}
$$

In the rest of this manuscript, the deviation of the signal from equilibrium is normalized in the range of $[0,1]$. Zero represents equilibrium, $M_{z}=M_{0}$ or $M_{+}=0$, whereas one denotes the farthest deviation from equilibrium as $M_{z}=$ $-M_{0}$ or $M_{+}=M_{0}$.

Brownstein and Tarr [15] proposed an eigenstate solution to the problem. A series of orthogonal spatial functions, $F_{1, n}(\mathbf{r})$, in the pore space that decay with time describe magnetization evolution:

$$
M_{z}(\mathbf{r}, t)=\sum_{n=0}^{\infty} A_{1, n} F_{1, n}(\mathbf{r}) e^{-t / T_{1, n}},
$$

where $A_{1, n}$ determines the relative contribution of each orthogonal spatial function that decays with relaxation time $T_{1, n} . F_{1, n}$ follows the terms of a generalized Fourier series based on cosines, Bessel functions of the first kind, and spherical Bessel functions of the first kind for symmetrical planar, cylindrical, and spherical geometries, respectively. Due to the orthogonality of eigenstates, each $F_{1, n}(\mathbf{r}) e^{-t / T_{1, n}}$ term satisfies Eqs. (1) and (3) with eigenvalues $1 / T_{1, n}$, such that $0<\cdots<T_{1,2}<T_{1,1}<T_{1,0}$.

By integrating the spatial functions, one can find the observed magnetization as a series of exponential functions that decay with time:

$$
m_{z}(t)=m_{z}(0) \sum_{n=0}^{\infty} I_{1, n} e^{-t / T_{1, n}} .
$$

Here $I_{1, n}$ are normalized relative intensities corresponding to eigenvalues and $m_{z}$ is the bulk magnetization. Equation (8), and its transverse-relaxation analog, describe multimodal features of relaxation decays, even for a single pore size.

Brownstein and Tarr [15] developed equations for $F_{1, n}$, $I_{1, n}$, and $T_{1, n}$ for planar, cylindrical, and spherical pores.
In such simple geometries, eigenvalues of a system of characteristic length $l$ are

$$
T_{i, n}=\frac{l^{2}}{4 D \xi_{i, n}^{2}},
$$

where $\xi_{i, n}$ are functions of the geometry of the system, eigenvalue number, and $N_{\mathrm{BT}, i} ; i=1$ and 2 for longitudinal and transverse relaxation, respectively. The BrownsteinTarr number,

$$
N_{\mathrm{BT}, i}=\frac{\rho_{i} l}{D},
$$

is a dimensionless number that compares the rate of surface relaxation to diffusion. The Brownstein-Tarr number is analogous to the Damköhler number of the second kind, $\mathrm{Da}^{\mathrm{II}}$, in chemical-reaction engineering, which is defined as the ratio of the chemical-reaction rate to mass-transfer rate and controls the overall rate of a surface reaction [34].

In a pore with planar geometry, $\xi_{i, n}$ are the positive roots of

$$
2 \xi_{i, n} \tan \xi_{i, n}=N_{\mathrm{BT}, i},
$$

which may be solved in subdomains of $\{0,[0+(1 / 2)-\varepsilon]$ $\pi$ \} for the ground eigenvalue, $n=0$, and

$$
\left[\left(n-\frac{1}{2}+\varepsilon\right) \pi,\left(n+\frac{1}{2}-\varepsilon\right) \pi\right],
$$

for the $n$th nonground eigenvalues, respectively. A sufficiently small $\varepsilon$, for example, $10^{-4}$, permits the numerical solution of Eq. (11) through standard bracketing rootfinding algorithms to find $\xi_{i, n}$. These dimensionless numbers will transform into eigenvalues through Eq. (9). It is usually sufficient to calculate only the ground eigenvalue and the first and second nonground eigenvalues, since the observation of higher eigenvalues is experimentally challenging.

A correct combination of pore size, $l$, and surface relaxivities, $\rho_{i}$, through numerical solutions of Eqs. (9)-(11) leads to a series of eigenvalues, $T_{i, n}$, along with relative intensities, $I_{i, n}$, that match the features of relaxation-decay measurements, $m_{z}(t)$ or $m_{+}(t)$. Guidelines on the detection of nonground eigenvalues are provided in Secs. III, $\mathrm{IV}$, and VI.

\section{A SIMPLIFIED PICTURE OF THEORY}

Porous materials consist of a solid matrix and pores. Consider a sandstone with pore spaces completely filled with an aqueous liquid phase. Surface-relaxation processes, including homonuclear dipole-dipole coupling, cross-relaxation by other nuclear spins, relaxation by paramagnetic ions, and relaxation by free electrons, enhance 
the relaxation rate of excited ${ }^{1} \mathrm{H}$ nuclei at the solid surface compared with that of bulk-liquid relaxation [11]. During a magnetic resonance relaxation experiment, excited protons diffuse from the bulk of the pores to the surfacebound layer, where they experience enhanced relaxation. The relaxed nuclei subsequently return to the bulk of the pores by diffusion, and the same process repeats for many protons until magnetization is completely relaxed. The diffusivity and surface relaxivity are important factors in this process, as displayed in Fig. 1, which shows a simplified picture of the process close to the surface-bound layer.

The importance of the relative magnitude of diffusivity and surface relaxivity are more easily observed farther from the surface-bound layer and by displaying magnetization, that is, analogous to chemical concentration, in a cross section of a pore. In a magnetic resonance relaxation experiment, magnetization is initially constant at all locations in the planar pore at time $t=0$, which is shown as $M_{z}(\mathbf{r}, 0)=1$ in Fig. 2 . With time, the relative magnitude of surface relaxivity, which is analogous to the surface reactivity in chemical-reaction engineering, and diffusivity affect the magnetization profile along the pore. If the diffusion of ${ }^{1} \mathrm{H}$ nuclei from the bulk of the pores to the pore surface is significantly faster than surface relaxation, nuclear magnetization in the pore fluid reduces homogeneously with time, as shown in Fig. 2(a). Otherwise, nuclear magnetization in the pore fluid diminishes inhomogeneously, as shown in Fig. 2(b). We demonstrate that magnetic resonance relaxation in geological porous materials is not fast enough to homogenize magnetization.
Therefore, the decay of magnetization in common experiments of magnetic resonance relaxation decay in rocks is more like that in Fig. 2(b) than the commonly assumed form of Fig. 2(a).

The inhomogeneous decaying magnetization is a summation of several eigenfunctions with relative factors of $A_{1, n}$, according to Eq. (7) s. The first three eigenfunctions of nuclear magnetization in the system of Fig. 2(b) are shown in Fig. 3. Nonground eigenvalues corresponding to oscillating spatial functions have corresponding short relaxation times. The nonground contributions, however, decay slowly enough to be detected in experiments. We demonstrate that it is possible to observe these nonground contributions with careful data acquisition and analysis.

Simplistic fast-diffusion models completely disregard nonground eigenvalues. The Brownstein-Tarr treatment, however, stipulates the existence of nonground eigenvalues in diffusion-relaxation processes, except in the limiting case of $N_{\mathrm{BT}, i} \rightarrow 0$, which is equivalent to the extreme case of fast diffusion. Therefore, given an appropriate signal-tonoise ratio (SNR) in measurements, it should be possible to observe nonground eigenvalue contributions in nonlimiting cases of $N_{\mathrm{BT}, i}>0$. For many practical samples, $N_{\mathrm{BT}, i}$ is far from the limiting case of zero, and the diffusion behavior is in, or close to, the intermediate-diffusion regime. Multimodal behavior is anticipated under these conditions for both $T_{1}$ and $T_{2}$. The important question is how to observe and identify these features. We take considerable care to prove the possibility and describe how. The presence of multimodal behavior in both $T_{1}$ and $T_{2}$ put forth

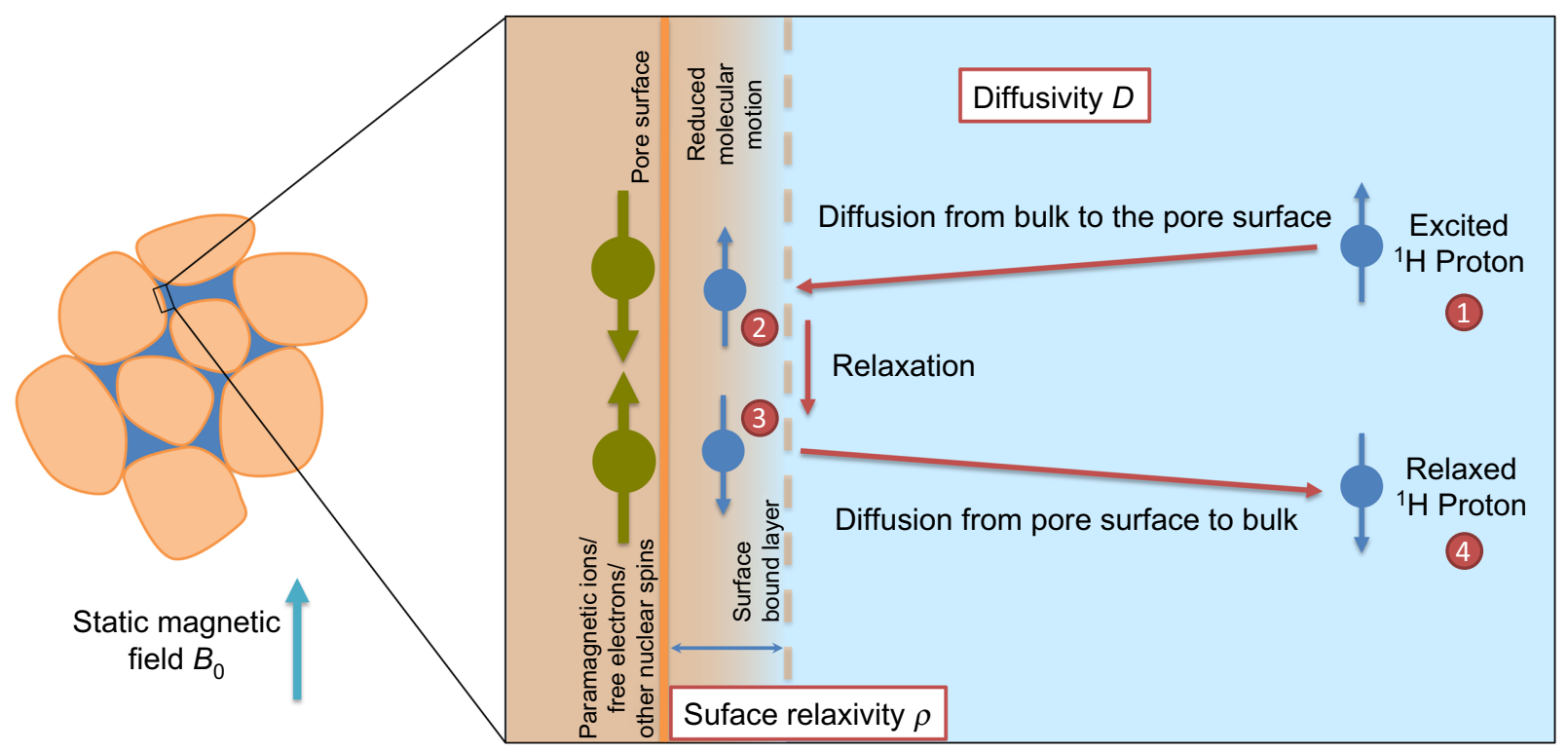

FIG. 1. Surface relaxation at the pore surface enhances magnetic resonance relaxation rate in a sandstone rock sample saturated with an aqueous phase. Excited ${ }^{1} \mathrm{H}$ nuclei (1) diffuse to the pore surface (2), where they relax by paramagnetic ions, free electrons, other nuclear spins, or homonuclear dipole-dipole coupling (3), and subsequently return to the bulk of the pore (4). This process is significantly affected by mass diffusivity and surface relaxivity. 

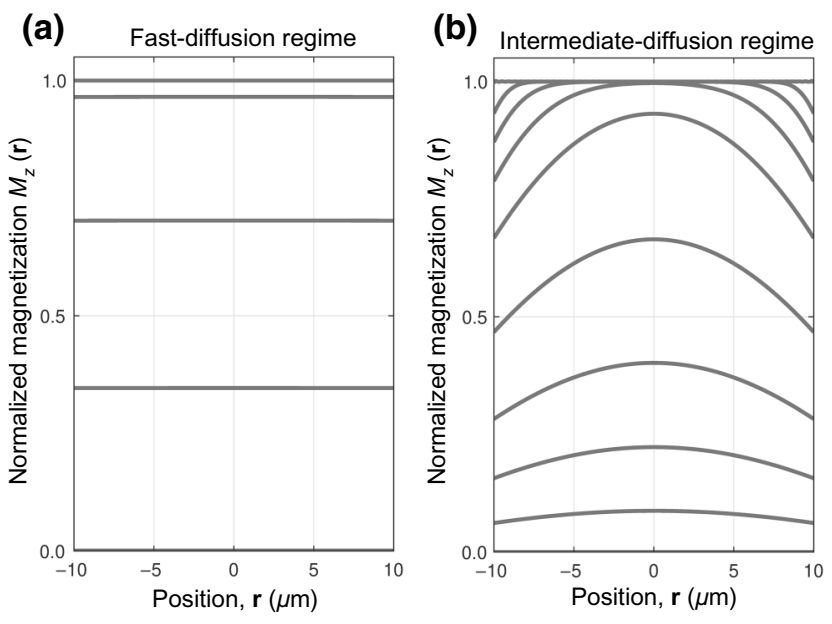

FIG. 2. Magnetization $M_{z}(\mathbf{r}, t)$ for (a) fast-diffusion regime, $N_{\mathrm{BT}, 1}=1.6 \times 10^{-3}, \quad \rho_{1}=0.2 \mu \mathrm{m} / \mathrm{s}$, and (b) intermediatediffusion regime, $N_{\mathrm{BT}, 1}=1.6, \rho_{1}=200 \mu \mathrm{m} / \mathrm{s}$. Magnetization profile reduces homogeneously for (a) fast-diffusion regime. Magnetization profile of (b) intermediate-diffusion regime, however, is the summation of several eigenfunctions with their corresponding relaxation times. In both cases, $l=20 \mu \mathrm{m}, T_{1 b}=$ $3 \mathrm{~s}$, and $D=2.5 \times 10^{-9} \mathrm{~m}^{2} / \mathrm{s} .50$ eigenvalues and 1000 profile points are used in numerical calculations. Magnetization profiles are for (a) $t=0,0.1,1,3$, and $20 \mathrm{~s}$ and (b) $t=0,0.0003,0.001$, $0.0032,0.01,0.032,0.06,0.1,0.16$, and $1 \mathrm{~s}$. First three eigenfunctions and eigenvalues of (b) are shown in Fig. 3. Video of Fig. 2(b) is available as Supplemental Material [35].

the $T_{1}-T_{2}$-relaxation correlation as a means to expand the parameter space to identify nonground eigenvalues more easily. It is easier to successfully understand the effect of nonground eigenvalues in one-dimensional measurements after considering the two-dimensional case.

\section{METHODS AND PARAMETERS}

Two-dimensional magnetic resonance relaxation methods have the potential to reveal complex fluid dynamics in porous media [36]. These measurements follow the same measurement periods as those in two-dimensional chemical exchange methods in magnetic resonance [37]: magnetization preparation, evolution, and detection. A period of mixing may also exist before detection, for example, in exchange-type experiments, such as $T_{2}-T_{2}$; it is, however, absent in correlation experiments, such as $T_{1}-T_{2}$.

The $T_{1}-T_{2}$ magnetic resonance relaxation correlation method [38,39] is composed of a $T_{1}$-encoding evolution segment and a CPMG $T_{2}$-encoding detection segment:

$$
\underbrace{\left[\pi-\tau_{1}-\frac{\pi}{2}\right]}_{\begin{array}{c}
T_{1} \text { encoding, } \\
\text { evolution }
\end{array}} \underbrace{\left[-\left(\tau_{i}-\pi-\tau_{i}\right)_{N}\right]}_{\begin{array}{c}
T_{2} \text { encoding, } \\
\text { detection }
\end{array}} .
$$

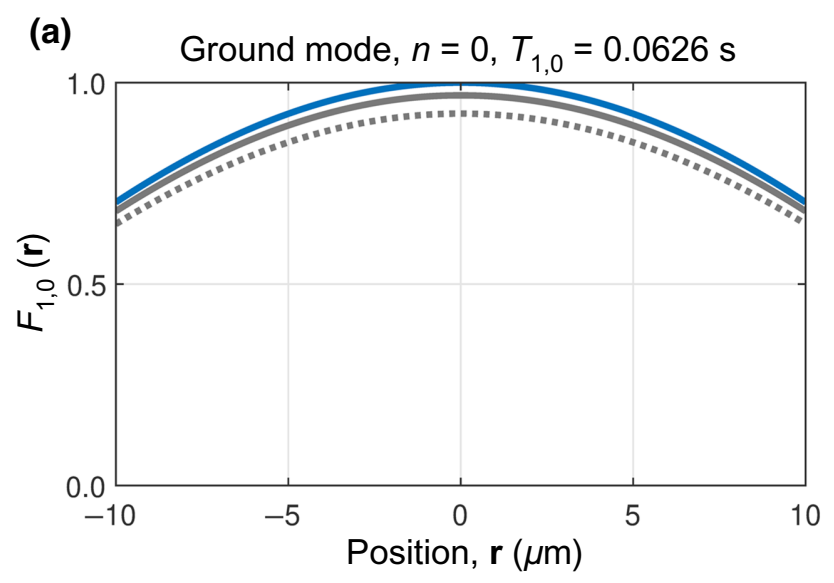

(b) First nonground mode, $n=1, T_{1,1}=0.0035 \mathrm{~s}$

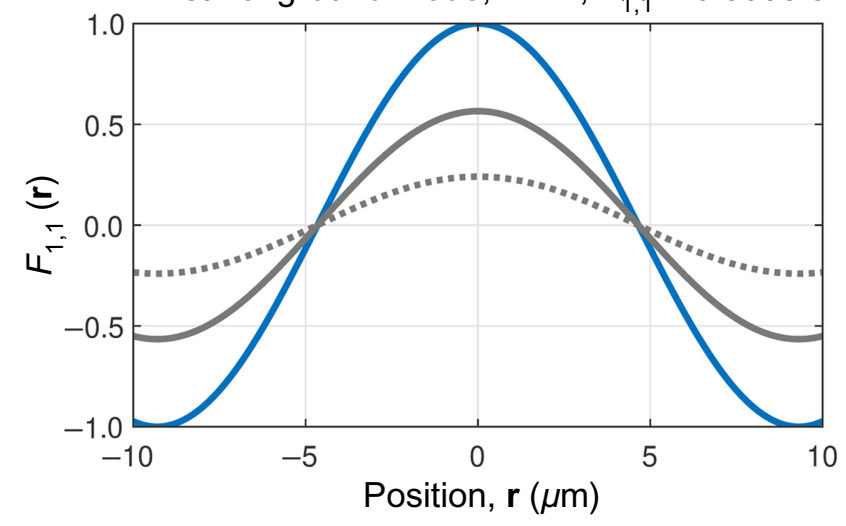

(c) Second nonground mode, $n=2, T_{1,2}=0.0010 \mathrm{~s}$

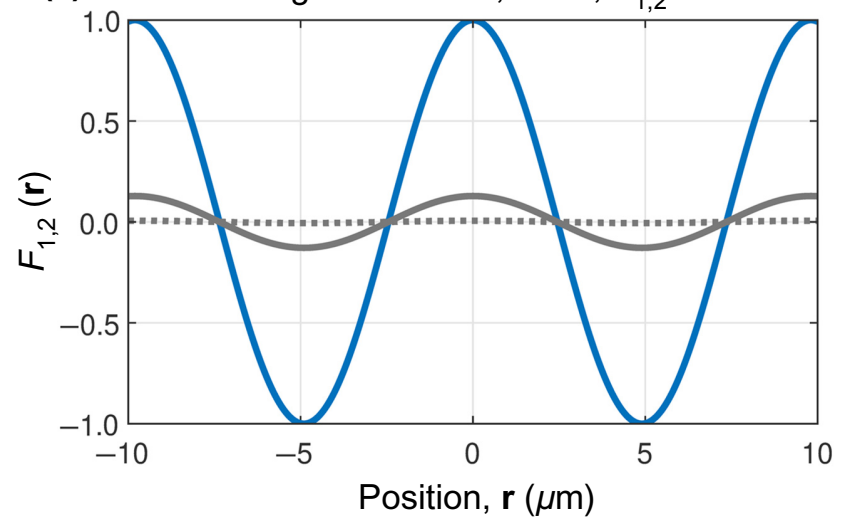

FIG. 3. First three eigenfunctions of the intermediate-diffusion regime system of Fig. 2(b). For the ground eigenstate $n=$ $0, \xi_{1,0}=0.7910, T_{1,0}=0.0626 \mathrm{~s}, A_{1,0}=1.1016, I_{1,0}=0.9903$; for the first nonground eigenstate $n=1, \xi_{1,1}=3.3744, T_{1,1}=$ $0.0035 \mathrm{~s}, A_{1,1}=-0.1282, I_{1,1}=0.0088$; and for the second nonground eigenstate $n=2, \xi_{1,2}=6.4074, T_{1,2}=0.0010 \mathrm{~s}, A_{1,2}=$ $0.0379, I_{1,2}=0.0007$. Gray dashed and dotted lines show relaxation of the eigenfunction at $t=2$ and $5 \mathrm{~ms}$, respectively. It is only at early times that it is possible to observe the contribution of nonground eigenvalues, as they relax much faster than the ground eigenvalue.

An inversion-recovery $T_{1}$ encoding is employed in this work, but saturation-recovery $T_{1}$ encoding [40,41], and 
even more complex encoding schemes [38], are also common and possible. Important parameters in the $T_{1}-T_{2}$ pulse sequence are described in the following. The echo spacing in the second segment is $2 \tau_{i}$. In experiments performed in this work, $\tau_{i}$ is set to 150,200 , or $300 \mu \mathrm{s}$ in different experiments. Experimental data are recorded as spin-echo signals at times $\tau_{2}=2 k \tau_{i}$, where $1 \leq k \leq N$ and $\tau_{2}$ is the time starting from the beginning of the $T_{2}-$ encoding segment. The experiment is repeated $P$ times, $P \geq 50$, with a nonlinear-spaced list of $\tau_{1}$ in the range of 0.0001 to $15 \mathrm{~s}$. The resonant frequency is reset for each repetition. Resetting the resonant frequency adjusts for a temperature-related field drift and results in superior relaxation-data quality, especially at short times. $N$ and $\tau_{i}$ are kept constant. $N$ has values between 4096 and 12288 in different samples, enough to accurately detect a baseline offset [42]. A conventional CPMG-phase cycling is used in the $T_{2}$-encoding segment with a phase shift of $90^{\circ}$ compared with other pulses in the $T_{1}$-encoding segment. Simple radio-frequency pulses are used, not composite pulses. Repetition delays of 15 to $25 \mathrm{~s}$ result in measurement times from less than $1 \mathrm{~h}$ to up to $6 \mathrm{~h}$.

The time-domain signal from the radio-frequency coil is recorded as a $P \times N$ matrix, $m_{+}\left(\tau_{1}, \tau_{2}\right)$,

$$
\begin{aligned}
m_{+}\left(\tau_{1}, \tau_{2}\right) & =\int_{\mathbf{r}} M_{+}\left(\mathbf{r}, \tau_{1}, \tau_{2}\right) d \mathbf{r} \\
& =\sum_{q=0}^{S} \sum_{p=0}^{R} I\left(T_{1, p}, T_{2, q}\right) e^{-\tau_{1} / T_{1, p}} e^{-\tau_{2} / T_{2, p}} .
\end{aligned}
$$

A regularized inverse two-dimensional (2D) Fredholm integral of the first kind transforms phase-corrected and normalized $m_{+}$into an $R \times S$ relaxation-correlation function, $I\left(T_{1, p}, T_{2, q}\right)$, to determine eigenvalues of magnetic resonance relaxation [43]. Phase correction consists of shifting the phase of complex-valued signals, so that they dominate the imaginary channel, similar to the zerothorder phase correction described by Mitchell et al. [44]. The imaginary channel is then normalized with respect to the maximum signal intensity. The Tikhonov regularization method of Venkataramanan et al. [45] is employed in this work and finds $I\left(T_{1, p}, T_{2, q}\right)$ as the solution to an optimization problem. The standard algorithm of Ref. [45] is applied in the inversion of relaxation data, but with careful selection of parameters for processing and viewing the results. Guidelines described in Sec. VI help to identity nonground eigenvalues from inverted relaxation data.

The regularization parameter $\alpha$ adjusts the smoothness of the solution by filtering out components of the solution that correspond to small singular values. In the inversion method of Venkataramanan et al., large values of $\alpha$ lead to smooth results, whereas a small $\alpha$ produces discretized solutions [45]. When $\alpha$ is very small, noise filtration is inadequate and the solution of $I\left(T_{1, p}, T_{2, q}\right)$ becomes oscillatory. On the other hand, when $\alpha$ is large, most components of the solution are also filtered out and $I\left(T_{1, p}, T_{2, q}\right)$ becomes overly smooth $[46,47]$. In this work, a series of $\log _{10}$-spaced regularization parameters, such as powers of ten in the range of 0.001 to 10000 , are used for data inversion to facilitate the correct detection of the ground and nonground eigenvalues. It is anticipated that ground eigenvalues dominate the solution and nonground eigenvalues will be absent when $\alpha$ is large. However, when $\alpha$ is small, nonground eigenvalues may be observed, but the ground eigenvalue may demonstrate peak splitting, an example of highly oscillatory behavior. The detection of nonground eigenvalues among low-intensity peaks is explained in Secs. VI and VII.

The consistency, reproducibility, and accuracy of our results are confirmed by performing experiments at three static magnetic fields with different magnets, consoles, and radio-frequency coils; processing data with two different 2D inversion software programs (that of Schlumberger [45] and another one developed in-house at the Centre for Oil and Gas - DTU of the Technical University of Denmark); repeating some of the measurements; and measuring similar sister samples.

Inversion-prepared spin-echo single-point imaging (INVSESPI) [48] as a one-dimensional phase-encoding $T_{1}-T_{2}$ imaging method is implemented in this work. The pulse sequence of the method is shown in Fig. 4 and is undertaken at $2.2 \mathrm{MHz}$. INVSESPI is composed of an inversion-recovery signal preparation with a spinecho single-point-imaging readout [49]. Phase-encoding trapezoidal gradient pulses between the $90^{\circ}$ pulse and the first refocusing $180^{\circ}$ pulse resolve position through Fourier transformation of the data to create an image. The gradient has a plateau duration of $200 \mu$ s with ramp-up and ramp-down durations of $100 \mu \mathrm{s}$. A maximum gradient of $0.78 \mathrm{G} / \mathrm{cm}$ is used with a resolution of 16 pixels, corresponding to a field of view of $80 \mathrm{~mm}$. The echo time is $2000 \mu \mathrm{s}$ and 1024 echoes are used with composite radio-frequency pulses of $45_{\pi / 2} 90_{0} 90_{3 \pi / 2} 45_{0}$ for a composite $90^{\circ}$ pulse and $180_{2 \pi / 3} 180_{4 \pi / 3} 180_{2 \pi / 3}$ for a composite $180^{\circ}$ pulse to compensate for the radio-frequency field inhomogeneity [49-51].

\section{MATERIALS AND INSTRUMENTS}

Samples studied in this work are Berea and Bentheimer sandstones acquired from Kocurek Industries (Caldwell, Texas, USA) and cut into cylindrical pieces of $38 \mathrm{~mm}$ in diameter and $50 \mathrm{~mm}$ in length. All samples are saturated with 2-mass-percent $\mathrm{NaCl}$ solutions, with $T_{1 b}=3.07 \mathrm{~s}$ and $T_{2 b}=2.80 \mathrm{~s}$ at $2.2 \mathrm{MHz}, T_{1 b}=2.82 \mathrm{~s}$ and $T_{2 b}=2.61 \mathrm{~s}$ at $8.5 \mathrm{MHz}$, and $T_{1 b}=2.62 \mathrm{~s}$ and $T_{2 b}=2.24 \mathrm{~s}$ at $98.8 \mathrm{MHz}$. The increasing ${ }^{1} \mathrm{H}$-relaxation rate of brine solution with the magnetic field intensity is hypothesized to be due to minor oxygen dissolution in the aqueous phase that occurs during 


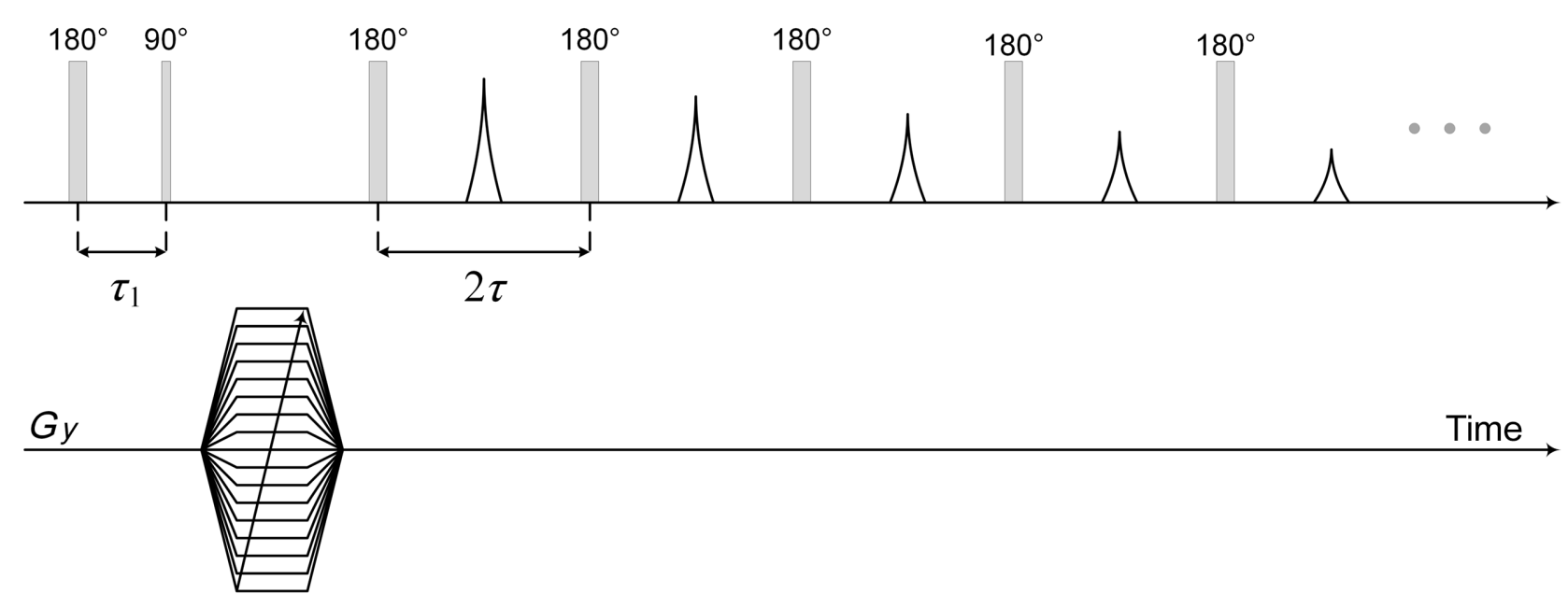

FIG. 4. INVSESPI method is a one-dimensional phase-encoding imaging variant of $T_{1}-T_{2}$ to measure confinement size in porous media.

sample preparation [52]. A water self-diffusivity of $D=$ $2.3 \times 10^{-9} \mathrm{~m}^{2} / \mathrm{s}$ at $25^{\circ} \mathrm{C}$ is used in all calculations [53]. Magnetic resonance experiments are undertaken with three different instruments: (a) an Oxford Maran Ultra verticalbore permanent magnet operating at a ${ }^{1} \mathrm{H}$ frequency of $2.2 \mathrm{MHz}$ with a $90^{\circ}$ pulse length of $27.5 \mu \mathrm{s}$, (b) an Oxford Maran DRX-HF vertical-bore permanent magnet operating at a ${ }^{1} \mathrm{H}$ frequency of $8.5 \mathrm{MHz}$ with a $90^{\circ}$ pulse length of $11 \mu \mathrm{s}$, and (c) a Nalorac 2.4-T horizontal-bore superconducting magnet equipped with a Tecmag console operating at a ${ }^{1} \mathrm{H}$ frequency of $98.8 \mathrm{MHz}$ with a $90^{\circ}$ pulse length of $12 \mu \mathrm{s}$. In-house software programs developed in MATLAB are used for data and image processing. The exponential analysis library provided by Schlumberger [45] is used in our data analysis.

Small subsamples are acquired for x-ray microtomography and SEM imaging. All x-ray microtomography measurements are undertaken with a Skyscan 1072 instrument and SEM imaging is undertaken with a JEOL JSM6400. Pore sizes from x-ray microtomography and SEM instruments are extracted by a skeleton mask of the distance transform in the pore space. Visualization of the pore spaces for Berea and Bentheimer are shown in Fig. 5.

\section{RESULTS}

According to the Brownstein-Tarr theory of magnetic resonance relaxation in porous materials, ground and nonground eigenvalues of relaxation are expected to be observed in the $T_{1}-T_{2}$-correlation plots: ground eigenvalues are observed as peaks of high intensity and nonground eigenvalues with peaks of lower intensity appearing at shorter relaxation times. Simulation and actual measurements are presented to demonstrate such features and the possibility of obtaining the average pore size.

\section{A. Simulations}

We assume reasonable pore parameters and show that the first nonground mode will be observed in a simulated experiment with a realistic signal-to-noise ratio by employing our regularization approach. Assume water with diffusivity $D=2.5 \times 10^{-9} \mathrm{~m}^{2} / \mathrm{s}$ and bulk longitudinal and transverse-relaxation-time constants of $T_{1 b}=$ $T_{2 b}=3 \mathrm{~s}$ in a planar geometry with pore size of $l=$ $20 \mu \mathrm{m}$ and longitudinal- and transverse-surface relaxivities of $\rho_{1}=200 \mu \mathrm{m} / \mathrm{s}$ and $\rho_{2}=300 \mu \mathrm{m} / \mathrm{s}$, respectively. This is consistent with the example previously presented in Sec. III. For this system, the time constants corresponding to the first three eigenvalues are $T_{1,0}=62.6 \mathrm{~ms}, T_{2,0}=$ $46.7 \mathrm{~ms}, T_{1,1}=3.5 \mathrm{~ms}, T_{2,1}=3.3 \mathrm{~ms}, T_{1,2}=1.0 \mathrm{~ms}$, and $T_{2,2}=1.0 \mathrm{~ms}$. Intensities of $0.982,0.0162$, and 0.0015 are assumed to correspond with the first three time constants. $T_{1}-T_{2}$-correlation plots for this system with a Gaussian noise of $\sigma_{\text {noise }}=0.002$ standard deviation, corresponding to a signal-to-noise ratio of 500, is shown in Fig. 6 . The (a)

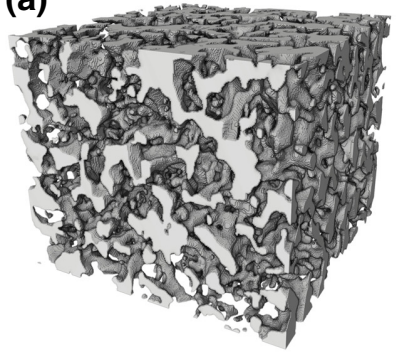

(b)

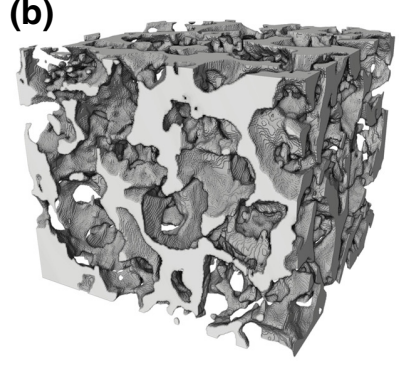

FIG. 5. Visualization of pore spaces in Berea (left) and Bentheimer (right) sandstones by x-ray microtomography. Boxes are $1000 \times 1000 \mu \mathrm{m}^{2}$ with a height of $860 \mu \mathrm{m}$. It is readily apparent that Bentheimer has a larger pore size than Berea. X-ray microtomography data are available from Ref. [54] and are described in Ref. [55]. 

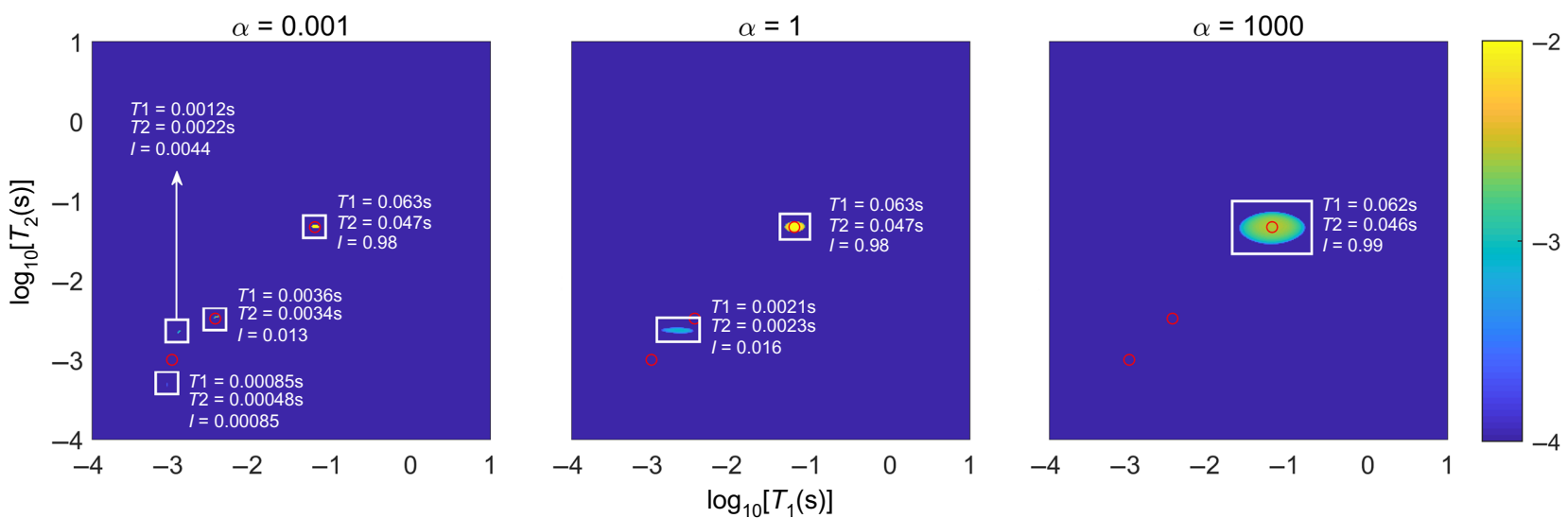

FIG. 6. $\log _{10}\left[I\left(T_{1, p}, T_{2, q}\right)\right]$ corresponding to the first three eigenvalues, $P 00, P 11$, and $P 22$, of a planar geometry with $l=10 \mu$ m, $\rho_{1}=200 \mu \mathrm{m} / \mathrm{s}, \rho_{2}=300 \mu \mathrm{m} / \mathrm{s}, D=2.5 \times 10^{-9} \mathrm{~m}^{2} / \mathrm{s}$, and $T_{1 b}=T_{2 b}=3 \mathrm{~s}$ with a Gaussian noise of $\sigma_{\text {noise }}=0.002$ at three regularization parameters of $\alpha=0.001,1$, and 1000. Intensity is logarithmically scaled in the range of $[-4,-2]$ and exact coordinates of contributing time constants are shown as red circles. No nonground eigenvalue is observed at $\alpha=1000$. At $\alpha=0.001$, the first nonground eigenvalue is observed very close to the theoretically exact coordinates, $\left(T_{1,1}, T_{2,1}\right)=(3.5 \mathrm{~ms}, 3.3 \mathrm{~ms})$. Second eigenvalue may not be observed, even at small regularization parameters, since its corresponding intensity is smaller than $\sigma_{\text {noise }}$. White boxes and text show the intensity contribution and logarithmic mean of the coordinates of observed peaks.

logarithmic intensity axis of Fig. 6, $\log _{10}\left[I\left(T_{1, p}, T_{2, q}\right)\right]$, facilitates the detection of low-intensity peaks, in contrast to linear color maps commonly employed in displaying relaxation-correlation data, $I\left(T_{1, p}, T_{2, q}\right)$. The actual positions of the first three eigenvalues are marked on the plot with red circles.

Three regularization parameters of $\alpha=0.001,1$, and 1000 vary the smoothness of the solution to demonstrate the possibility of the observation of the first nonground eigenvalue at its correct position at small $\alpha$. At $\alpha=1000$, the ground eigenvalue is observable as a wide peak, while nonground eigenvalues are not detectable. At a sufficiently small $\alpha$, such as $\alpha=1$, the smoothness condition is such that the first nonground eigenvalue appears in the data as a small intensity peak marked very close to the correct position. A smaller regularization parameter moves the first nonground eigenvalue in the inverted data to its actual value. The second nonground eigenvalue is still not observed at $\alpha=0.001$, since its corresponding intensity, 0.0015 , is smaller than the assumed Gaussian noise, $\sigma_{\text {noise }}$. Back calculation of the pore size and relaxivity parameters from the simulation experiments results in $l=20.3 \mu \mathrm{m}$, $\rho_{1}=197 \mu \mathrm{m} / \mathrm{s}$, and $\rho_{2}=292 \mu \mathrm{m} / \mathrm{s}$; these values are very close to the set parameters. Richer behavior of nonground eigenvalues may be observed in complex porous media, such as sedimentary rocks, where small regularization parameters necessary to show nonground eigenvalues lead to peak splitting, or pearling, of the ground eigenvalue.

\section{B. Two-dimensional experiments}

A Berea sandstone core plug is examined by the $T_{1}-T_{2}$-correlation method at $8.5 \mathrm{MHz} . I\left(T_{1, p}, T_{2, q}\right)$ for
Berea with different regularization parameters of $\alpha=100$, 1 , and 0.01 is shown in Fig. 7. At $\alpha=100$, only the ground eigenvalue, $P 00$, is observed. Detection of the ground eigenvalue is straightforward: at large $\alpha$, such as $\alpha=100$, if there is only one peak present, the porous medium is unimodal and the mode of the peak is used to determine ground eigenvalues, $T_{1,0}$ and $T_{2,0}$. The position of the mode of this peak, but not its magnitude, is stable at regularization parameters of any magnitude.

The detection of nonground eigenvalues is more challenging due to their low intensity. The intensity of $\log _{10} I$, instead of $I$, is displayed to enhance the visibility of nonground eigenvalues. The range of $\log _{10} I$ displayed is limited to $[-4,-2]$ to filter out possible peaks of very small amplitude that may be produced due to the limited SNR. We use the variation of the regularization parameter to aid detection of nonground eigenvalues. This is in contrast with common exponential analysis, where a single appropriate regularization parameter is sought. $\log _{10} I$ at small $\alpha$ is likely to reveal the first and even second nonground eigenvalues of relaxation. The first and second nonground eigenvalues of the diffusion-relaxation equation, $P 11$ and $P 22$, emerge as peaks of small intensity at small $\alpha=0.01$, as shown in Fig. 7 and in the Supplemental Material [35].

Two complications arise with reducing $\alpha$ in $T_{1}-$ $T_{2}$ data: (a) pearling and (b) peaks of small amplitude other than nonground eigenvalues; the former is very pronounced, while the latter takes place in complex natural samples and experiments with a limited SNR. These two effects increase the complexity of detecting nonground eigenvalues of relaxation. The significance of each complication is discussed below. 
Pearling, or peak splitting, is a common problem inherent in the numerical inversion of Fredholm integrals of the first kind [56]. A large peak splits into a number of smaller peaks at small $\alpha$, as shown in Fig. 7 for $\alpha=0.01$. Small peaks of a pearled ground eigenvalue may hinder the detection of nonground eigenvalues. Pearling is only a problem for the ground eigenvalue peak. The pearled peaks appear within the domain of the ground peak and can be identified from a series of correlation maps with $\operatorname{logarithmically}$ decreasing $\alpha$. The position of the mode of the nonground eigenvalue peak is stable at regularization parameters of any magnitude (see the Supplemental Material [35]). Pearling does not affect nonground peaks due to their small domain.

Although the multicomponent behavior of relaxation maps is the genuine reflection of the measured relaxationtime distributions [57], peaks other than those described in Sec. II may appear in $T_{1}-T_{2}$-relaxation-correlation maps. These peaks may be due to problems associated with data inversion or a limited SNR, or they may bear useful information on exchange, water associated with surface and clays, and water molecules that do not relax at pore surfaces. Regardless of the origin of these peaks, their presence complicates the detection of nonground eigenvalues. A series of guidelines for displaying data and searching for nonground eigenvalues, however, simplifies the detection of nonground eigenvalues. The display of the logarithm of intensity only in the range of $[-4,-2]$, in particular, removes peaks of very small intensity that may appear due to a low signal-to-noise ratio. A complete description of magnetic resonance relaxation in porous media with all possible fluid-dynamic effects will help to identify minor peaks with a physical origin.

Nonground eigenvalues of the relaxation-diffusion system are detached from the ground eigenvalue and have a well-defined amplitude and position with respect to that of the ground eigenvalue. The first nonground relaxation-time constant is approximately one tenth of the ground relaxation-time constant, while the intensity ratio is between 0.02 and 0.10, according to the Brownstein-Tarr theory (see Figs. 1 and 2 in Ref. [15]). For both ground and nonground eigenvalues, the eigenvalues approximately follow the diagonal line and $T_{2} \leq T_{1}$. The correct selection of eigenvalues leads to a cluster of peaks that will match the theoretical predictions of Brownstein and Tarr for simple geometries through optimization of pore size $l$, longitudinal-surface relaxivity $\rho_{1}$, and transverse-surface relaxivity $\rho_{2}$. If selected peaks fail to match experimental results with sensible optimized parameters, a different set of peaks is selected as the nonground eigenvalue and optimization is repeated.

A direct-search method [58] varies $\log _{10}[l(\mathrm{~m})]$, $\log _{10}\left[\rho_{1}(\mathrm{~m} / \mathrm{s})\right]$, and $\log _{10}\left[\rho_{2} /(\mathrm{m} / \mathrm{s})\right]$, all in the range of $[-7,-3]$, to minimize the difference between the ground and nonground transverse- and longitudinal-relaxation eigenvalues between those calculated from Eqs. (9)-(11) and those of measurements. This method, at $8.5 \mathrm{MHz}$, calculates a pore size of $54 \mu \mathrm{m}$, in comparison with values of 26 and $39 \mu \mathrm{m}$ from SEM and x-ray microtomography, respectively.

The robustness of our procedure and conclusions are examined by measuring similar Berea core plugs in different magnetic fields. This activity is believed to demonstrate (1) the wide applicability of the method to different instruments, (2) the effect of internal magnetic field gradients, and (3) the robustness of the method. Similar Berea core plugs are measured in three different magnetic fields of 2.2, 8.5, and $98.8 \mathrm{MHz}$ with SNRs of 189, 1240, and 11300 , respectively. The results of these experiments are shown in Fig. 8 and tabulated in Tables I and II. The main peaks
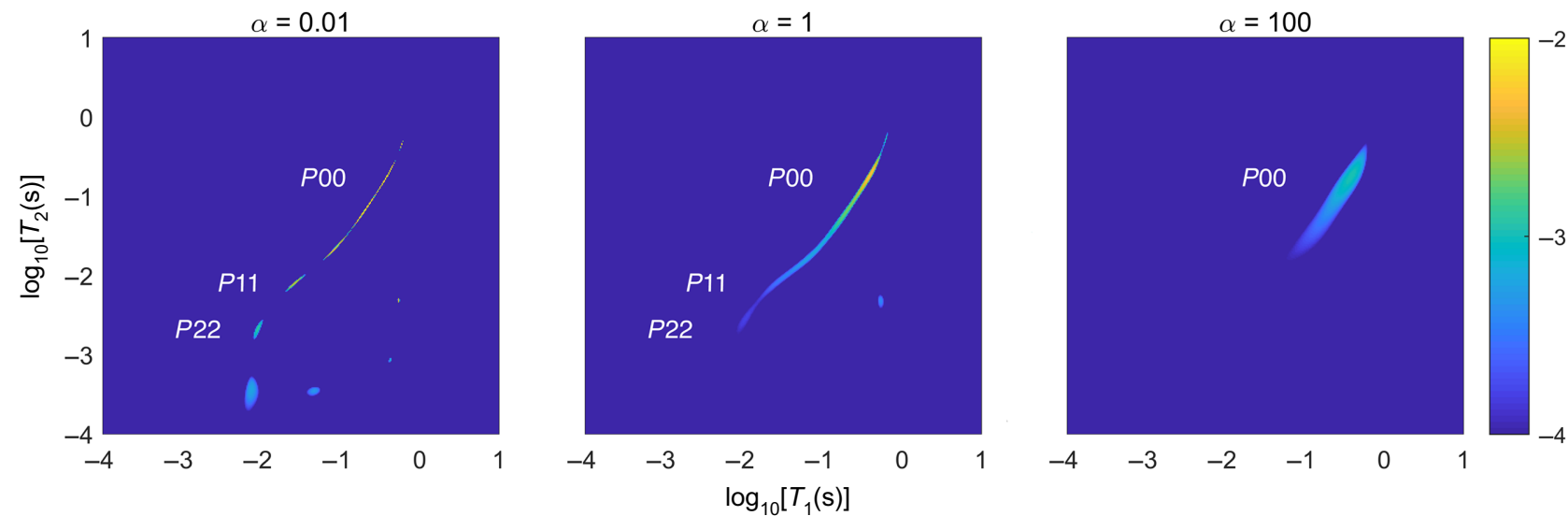

FIG. 7. $\log _{10}\left[I\left(T_{1, p}, T_{2, q}\right)\right]$ from $T_{1}-T_{2}$-correlation map of a Berea sandstone sample at $8.5 \mathrm{MHz}$ with three regularization parameters $\alpha=0.01,1$, and 100. Only the ground eigenvalue, $P 00$, is observed at $\alpha=100$. Ground eigenvalue peak pearls, splits into smaller peaks, at $\alpha=0.01$. It is possible to observe nonground eigenvalues $P 11$ and $P 22$ at sufficiently small regularization parameter of $\alpha=0.01$ (see the Supplemental Material [35]). Relative intensities of $P 00, P 11$, and $P 22$ peaks are $0.831,0.0728$, and 0.0473 , respectively. 
(a)

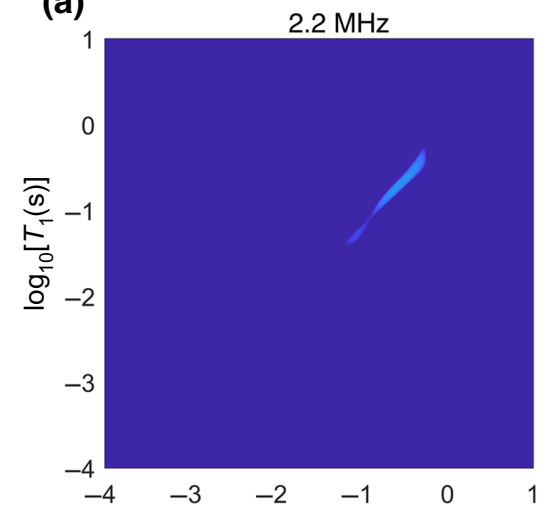

(b)

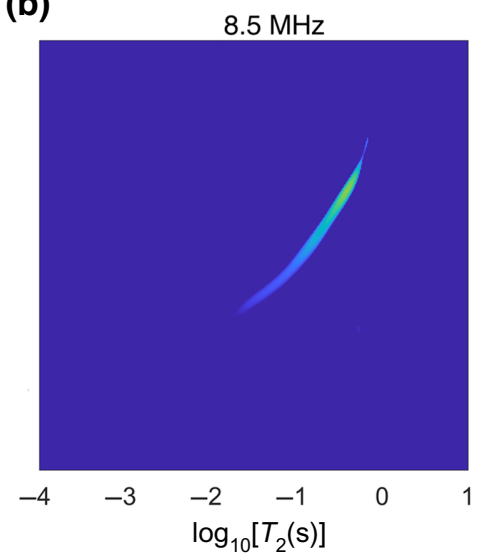

(c)

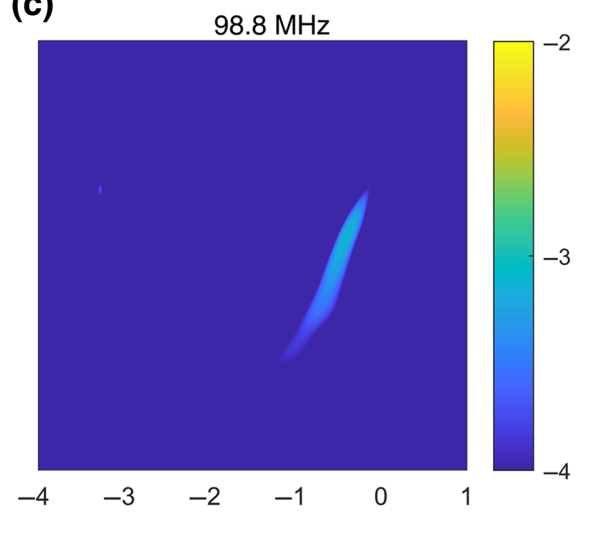

FIG. 8. $T_{1}-T_{2}$-correlation map of Berea sandstone at (a) 2.2, (b) 8.5 , and (c) $98.8 \mathrm{MHz}$; all values are normalized before inversion and inverted with a regularization parameter of $\alpha=10$. Slope of the ground eigenvalue changes from one at $2.2 \mathrm{MHz}$ to two at 98.8 MHz. This change in slope is due to fast transverse relaxation at $98.8 \mathrm{MHz}$ due to high surface relaxivity or internal gradients. Similar pore sizes are calculated from three measurements. $T_{1}-T_{2}$-correlation map of Berea sandstone at 2.2 and $98.8 \mathrm{MHz}$ may be found in Ref. [30] and the Supplemental Material [35], respectively.

in the $T_{1}-T_{2}$-correlation plots of Fig. 8 have a narrow pattern, which indicates a high correlation between $T_{1}$ and $T_{2}$ at all three magnetic fields. The slope of the ground eigenvalue changes from a value of one at $2.2 \mathrm{MHz}$ to two at $98.8 \mathrm{MHz}$. Transverse relaxation is significantly faster than diffusion at $98.8 \mathrm{MHz}$. Under this condition, a distinct slope of two in the $\log _{10}-\log _{10} T_{1}-T_{2}$ plot is observed, which suggests that $T_{2,0} \propto l^{2}$, while $T_{1,0} \propto l$. The quadratic relationship for transverse relaxivity may be due to internal magnetic field gradients, to the slow-diffusion regime, or both. We will investigate the main cause of this feature in a future work. Similar pore-size values are acquired using this method for Berea at 2.2, 8.5, and 98.8 MHz: 49, 54 , and $56 \mu \mathrm{m}$, for pore size, and 90,85 , and $90 \mu \mathrm{m} / \mathrm{s}$ for longitudinal-surface relaxivity, respectively. Experimental and numerical values do not match for the transversesurface relaxivity of Berea at $98.8 \mathrm{MHz}$, probably due to the strong effect of magnetic field gradients not considered in our method. Only $T_{1}$ is matched at this magnetic field. This method predicts transverse-surface-relaxivity values

TABLE I. Time constants of longitudinal and transverse magnetic resonance relaxation in Berea sandstone at three static magnetic fields.

\begin{tabular}{lcccc}
\hline \hline Eigenvalue & $\begin{array}{c}\text { Time } \\
\text { constants } \\
(\mathrm{ms})\end{array}$ & $2.2 \mathrm{MHz}$ & $8.5 \mathrm{MHz}$ & $98.8 \mathrm{MHz}$ \\
\hline Ground & $T_{1,0}$ & 322 & 368 & 369 \\
& $T_{2,0}$ & 237 & 169 & 46.9 \\
First & $T_{1,1}$ & 22.3 & 26.5 & 29.0 \\
$\quad$ nonground & $T_{2,1}$ & 16.8 & 8.0 & 0.6 \\
Second & $T_{1,2}$ & 5.9 & 9.1 & 5.4 \\
$\quad$ nonground & $T_{2,2}$ & 3.8 & 2.2 & 0.5 \\
\hline \hline
\end{tabular}

of 150 and $435 \mu \mathrm{m} / \mathrm{s}$ for Berea at 2.2 and $8.5 \mathrm{MHz}$, respectively. Deviations from model predictions are observed in the transverse-relaxation dimension, which is likely to be due to the effect of magnetic field gradients. These deviations are correlated to the applied magnetic field intensity; compare the positions of nonground peaks in Fig. 7 versus Fig. 2 in Ref. [30], Table I, for Berea sandstone. No deviation from model predictions is observed in the longitudinal-relaxation dimension. This work employs a more refined calculation procedure and reports larger pore size and smaller surface relaxivity values than those of Ref. [30].

In contrast to rocks with unimodal pores, if at large $\alpha I\left(T_{1, p}, T_{2, q}\right)$ displays two peaks, the sample has a bimodal pore-size distribution. In the case of bimodal ground eigenvalues, the amplitude of the peaks displays their relative volumetric size, and the connection of two such peaks demonstrates the possibility of exchange between geometric domains. Bentheimer sandstone demonstrates such bimodal ground eigenvalues, as shown in Fig. 9 and Table III. Two ground eigenvalues,

TABLE II. Average pore size and surface relaxivities of Berea sandstone measured at three static magnetic fields as well as using $\mathrm{x}$-ray microtomography (XMT) and scanning electron microscopy (SEM).

\begin{tabular}{lccccc}
\hline \hline Parameter & $2.2 \mathrm{MHz}$ & $8.5 \mathrm{MHz}$ & $98.8 \mathrm{MHz}$ & XMT & SEM \\
\hline$l(\mu \mathrm{m})$ & 49 & 54 & 56 & 39 & 26 \\
$\rho_{1}(\mu \mathrm{m} / \mathrm{s})$ & 90 & 85 & 90 & - & - \\
$\rho_{2}(\mu \mathrm{m} / \mathrm{s})$ & 150 & 435 & $\mathrm{a}$ & - & - \\
\hline \hline
\end{tabular}

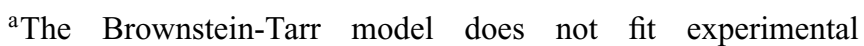
transverse-relaxation data at $98.8 \mathrm{MHz}$. 
TABLE III. Time constants of longitudinal and transverse magnetic resonance relaxation in large and small pores of Bentheimer sandstone at $2.2 \mathrm{MHz}$.

\begin{tabular}{lccc}
\hline \hline Eigenvalue & $\begin{array}{c}\text { Time } \\
\text { constants } \\
(\mathrm{ms})\end{array}$ & Large pores & Small pores \\
\hline Ground & $T_{1,0}$ & 1310 & 199 \\
First nonground & $T_{2,0}$ & 995 & 106 \\
& $T_{1,1}$ & 52 & 9.6 \\
& $T_{2,1}$ & 44 & 6.2 \\
\hline \hline
\end{tabular}

$L 00$ and $S 00$, are observed with an approximate intensity ratio of 12 . The large pore environment pertains to intergrain porosity and small pores are associated with porosity in clay regions. Pore-size calculations from eigenvalues predict 71 and $31 \mu \mathrm{m}$ for large and small pores, respectively. Surface relaxivities are $\rho_{1}=17$ and $\rho_{2}=26 \mu \mathrm{m} / \mathrm{s}$ for large pores and $\rho_{1}=89$ and $\rho_{1}=216 \mu \mathrm{m} / \mathrm{s}$ for small pores. The SNR of the 2D time-domain data for Bentheimer is 218. X-ray microtomography demonstrates a pore-size distribution for Bentheimer in the range of 6.5 to $126 \mu \mathrm{m}$ with a mode of $42 \mu \mathrm{m}$, while the pore-size distribution obtained from SEM is in the range of 1 to $86 \mu \mathrm{m}$ with a mode of $38 \mu \mathrm{m}$; both sets of results confirm the magnetic resonance pore sizing method for Bentheimer sandstone, as shown in Fig. 10. Microtomography measurements, with a resolution of $2.7 \mu \mathrm{m}$, do not demonstrate bimodal pore-size distributions; however, SEM shows pore-size distributions connected to the dominant peak in the range of 1 to $14 \mu \mathrm{m}$.

The shape of the SEM and microtomography pore-size distributions of Berea and Bentheimer sandstones, shown in Fig. 10, may appear similar to one another. There are, however, two distinct differences: first, the pore-size distributions of Berea sandstone shift to smaller pore sizes, in comparison with those of Bentheimer (also see Fig. 5); second, both SEM and microtomography pore-size distributions of Bentheimer are more negatively skewed than Berea sandstone, indicating the population of pores of small sizes [55]. Despite the apparent similarities between the pore-size distributions of Berea and Bentheimer in Fig. 10, the large contrast between the $T_{1}-T_{2}$-relaxationcorrelation plots of Figs. 7 and 9 demonstrates the strong effect of not only the pore-size distribution, but also surface relaxivity of grains and exchange between water in the bulk of the pores and that close to or inside clay agglomerates.

\section{Spatially resolved 2D relaxation correlation}

A spatially resolved variant of the pore-sizing method measures the confinement size of brine during steady-state imbibition in Berea. Confinement size here refers to a characteristic length of brine clusters in partially saturated pores, in contrast to the pore size that describes the characteristic length of fully brine-saturated pores. Two-mass-percent $\mathrm{NaCl}$ solution is injected through tubing into an air-saturated Berea core plug sealed with a Viton sleeve and heat-shrink tubing and restricted by Teflon endcaps. The core plug is vertical in the magnet and the direction of brine flow is upwards. As expected, a saturation variation develops in the vertical core plug during a slow flood, in agreement with classical experiments reported by Geffen et al. [59]. This experiment simulates water injection or water ingress into an underground gas reservoir. In such systems, the volumetric fraction of rock-pore space occupied by the gas phase increases with
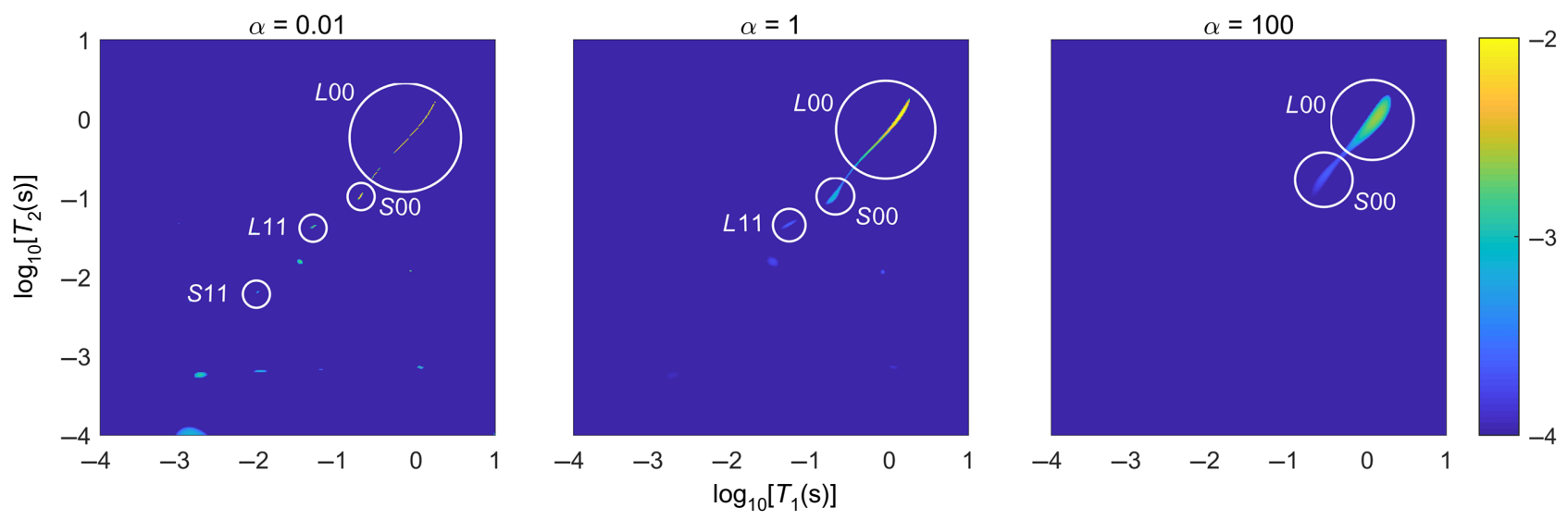

FIG. 9. $T_{1}-T_{2}$-correlation map of a Bentheimer sandstone sample at $2.2 \mathrm{MHz}$ with three regularization parameters, $\alpha=0.01,1$, and 100. $L p p$ and Spp, respectively, refer to eigenvalues of relaxation for large and small pores. Large pores are associated with intergrain porosity and small pores are associated with water in feldspar fragments and clay regions. Volume of water in the intergrain space is approximately 12 times that of the volume of water associated with feldspar fragments and clays. Nonground eigenvalues are marked with white circles of L11 and S11. See the Supplemental Material [35] for the $T_{1}-T_{2}$-correlation map as a function of $\alpha$. 

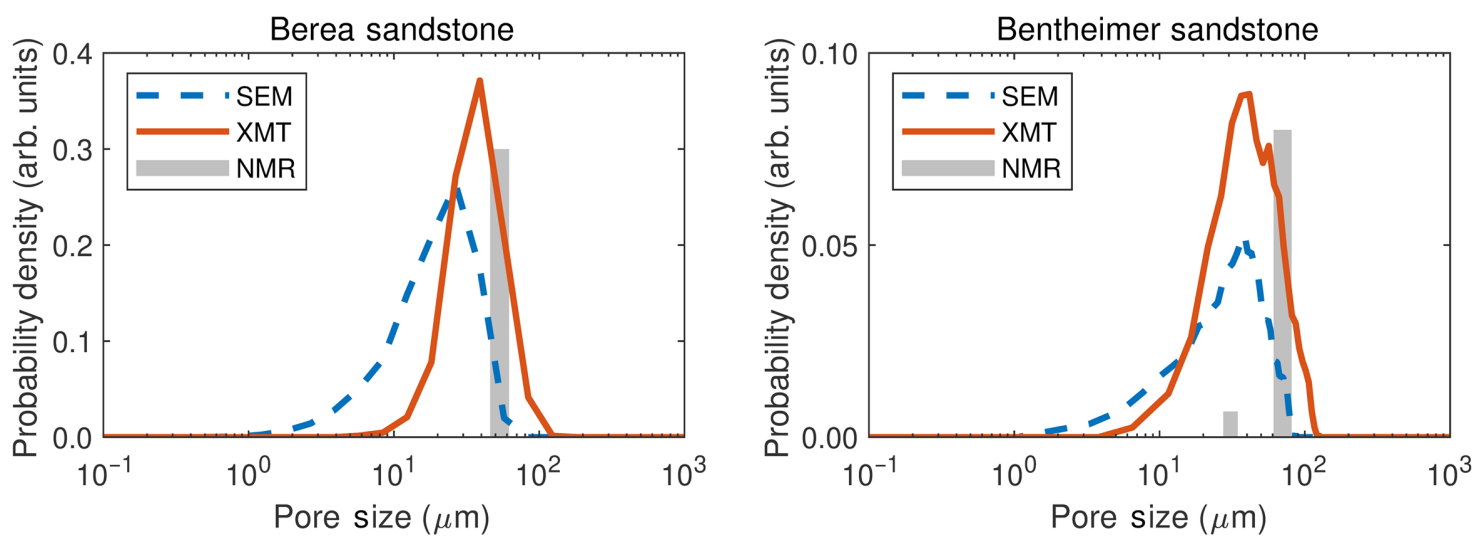

FIG. 10. Volumetric probability density of pore diameters obtained from SEM (dashed line) and XMT (solid line) for Berea (left) and Bentheimer (right) sandstones. Pore diameters from magnetic resonance measurements are shown as gray rectangles (NMR), with widths showing the perceived uncertainty and height showing the relative volume of each pore size for Bentheimer sandstone.

height. Since the water phase preferentially wets the rock surface and dominates the pore volume, a smaller waterconfinement size is expected to be associated with higher gas-phase volumetric fractions [60].

INVSESPI, the spatially resolved phase-encoding $T_{1}-$ $T_{2}$ method, imaged the core plug with 16 gradient values corresponding to 16 imaging points. Figure 11 demonstrates the confinement size of the water phase along the core plug. At each image pixel, a $T_{1}-T_{2}$-correlation function is acquired, nonground eigenvalues are identified, and the confinement size is computed according to Eqs. (9)-(11). It is possible to determine the nonground eigenvalue in all but one of the $T_{1}-T_{2}$ maps within the core plug. Water-confinement size decreases with height from the bottom of the core plug, in agreement with expectations. The relatively long echo time of the imaging method, $2 \mathrm{~ms}$, may be a limiting factor for some samples.

\section{One-dimensional relaxation}

The observation of nonground eigenvalues of magnetic resonance relaxation is also possible in $T_{2}$ or $T_{1}$ distributions measured by CPMG and inversion-recovery methods, which are important in petroleum laboratory measurements, well-logging, and industrial measurement and control. It is usually assumed that nonground eigenstates are not observed in $T_{1}$ and $T_{2}$ distributions and that these distributions are direct proxies for pore size. Here, we demonstrate that this is not the case and estimate the
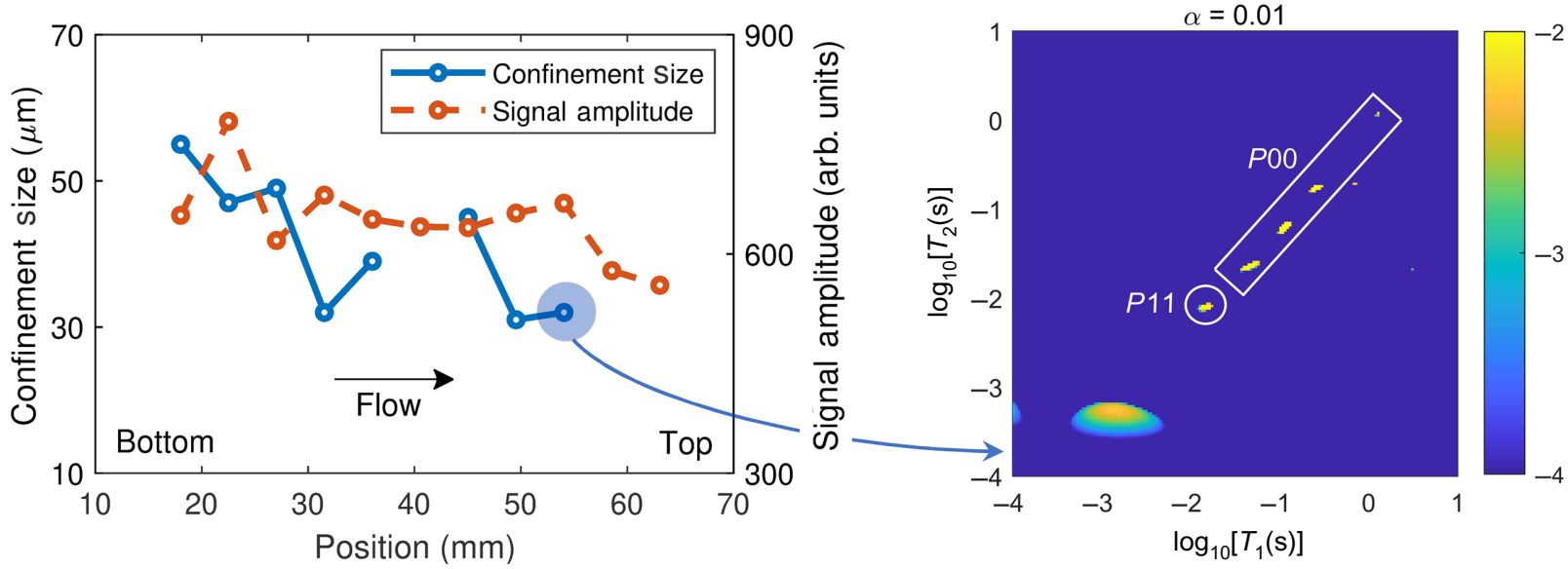

FIG. 11. Confinement size (solid line) and signal amplitude (dashed line) in a Berea core plug undergoing the imbibition process (left) and the $T_{1}-T_{2}$-relaxation-correlation map of the sample at a position of $54 \mathrm{~mm}$. Water is injected from the bottom of the core plug, at small position values. Decreasing apparent pore size is observed due to partial saturation of pores closer to the top side of the core plug. At data discontinuity, the method is unable to detect the nonground eigenvalue and measure the confinement size. Markers show measurement quantities, while the lines are a guide for the eye. 


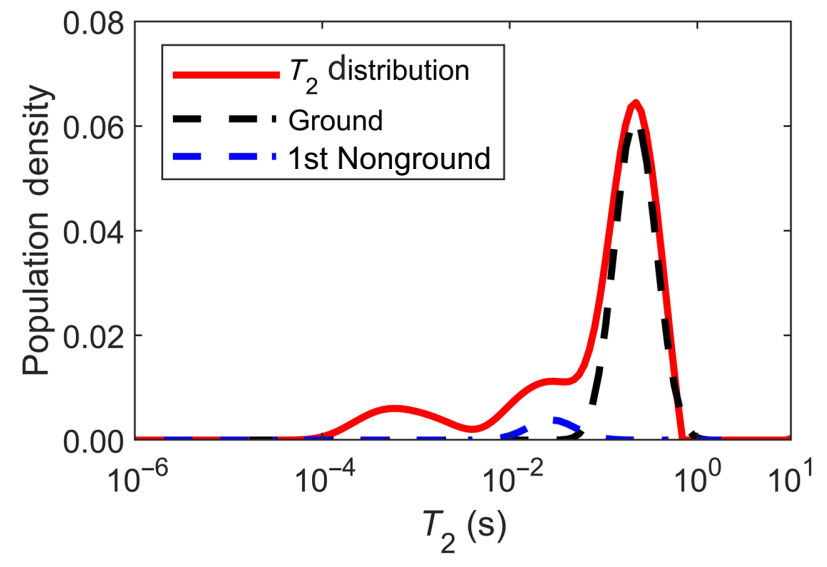

FIG. 12. $\quad T_{2}$ distribution of Berea sandstone and its estimated ground and nonground eigenvalues at $2.2 \mathrm{MHz} . T_{2}$ distribution (solid line) is measured using the CPMG method with an interecho spacing of $300 \mu \mathrm{s}$. Varying $l$ and $\rho_{2}$ and using a planar geometry for solving eigenvalues of the relaxation-diffusion equation lead to estimated (dashed line) contributions to the $T_{2}$ distribution by the dominant ground eigenvalues and a smaller nonground eigenvalue peak. Shortest relaxation-time peak is ascribed to surface water in the roughness of the pores and clay agglomerates.

pore size for unimodal-pore-size Berea sandstone from a single $T_{2}$ distribution, as shown in Fig. 12. The pore diameter of Berea sandstone is estimated to be $59 \mu \mathrm{m}$, with a distribution in the range of 20 to $155 \mu \mathrm{m}$ with $\rho_{2}$ of $300 \mu \mathrm{m} / \mathrm{s}$.

Similar attempts to measure pore size from relaxation decays have been previously made by Davies et al. [20,21] without clearly identifying nonground eigenvalues. The $T_{1}-T_{2}$ method, unlike one-dimensional (1D) relaxation, makes it easier to separate relaxation peaks for bimodal and multimodal pore-size distributions. This is especially true given the effects of internal magnetic field gradients previously pointed out in this work. Therefore, it is very unlikely that the ground eigenvalues of one pore size exactly match nonground eigenvalues of another pore size with similar intensity in $T_{1}-T_{2}$ experimental results. In the remote possibility of such an event, those pores should have a very small volume fraction to match nonground features of a larger pore and are hence inconsequential from a scientific or engineering standpoint. Pore-size measurements from 1D relaxation data of samples with a wide range of pore sizes might only have limited applicability. Prior information about the sample, such as its surface relaxivity, and acquiring 1D relaxation data with a high SNR may extend this method to pore sizing of complex samples from 1D data.

\section{DISCUSSION}

Song et al. [36] previously suggested that common assumptions in magnetic resonance relaxation of porous materials might break down in two cases of (1) sufficiently large surface relaxation within a given pore that involves several eigenvalues, and (2) interpore diffusion, also referred to as diffusive coupling. This work addresses the former and is complementary to the latter. Diffusive coupling leads to complex diffusion behavior that may be observed in the $T_{1}-T_{2}$ method as negative peaks at short $T_{1}$ and long $T_{2}[36,62]$. These peaks may not be identified by common $2 \mathrm{D}$ relaxation-inversion methods that employ non-negative optimization routines [36]. The effects of diffusive coupling in the case of bimodal ground eigenvalues may be observed as a connection of two such peaks, which demonstrates the possibility of exchange between two geometric domains. Diffusive coupling between two pore-size groups does not interfere with the nonground eigenvalues of diffusion relaxation discussed in this work. Even in the presence of diffusive coupling, it is still possible to observe small peaks of nonground eigenvalues of simple relaxation approximately along a diagonal line in $T_{1}-T_{2}$-correlation experiments [see Figs. 3(c) and 4(c) in Ref. [61] ].

Surface-relaxivity values reported in this work are systematically higher than those reported in the literature based on comparing relaxation size distributions with independent pore-size measurements using microscopy or gas adsorption [29]. However, our reported results are closer to those assumed by Davies et al. [20,21] and measured by Müller-Petke and co-workers [25,27], who used Brownstein-Tarr solutions. The systematic difference between surface relaxivities is because (a) the planar geometry assumed in this work provides less surface area per volume compared with a spherical geometry; (b) the numerical solution of equations is more sensitive to the pore size than surface relaxivity; and, most important of all, (c) there is a fundamental difference between the physics of fast-exchange systems and Brownstein-Tarr solutions that lead to surface-relaxivity values that should not necessarily have the same values. Surface-relaxivity values predicted using this method, therefore, should not be used in the two-site fast-exchange models of relaxation in porous media.

The Brownstein-Tarr theory of magnetic resonance relaxation in porous materials expects a ground relaxation time that is linear with pore size and nonground relaxation times that are quadratic with pore size in the fast-diffusion regime. These approximations are not exactly valid due to the intermediate-diffusion regime, commonly observed in magnetic resonance relaxation in porous materials (see Fig. 1 in Ref. [15]), and even if true may not be easily observed in plots of logarithmic intensity bound in the range of $[-4,-2]$. Regardless, the spread of the ground eigenvalue peak is substantially larger than that of nonground eigenvalue peaks in all measurements performed in our laboratory. Even in simulations, we observe a larger spread in the ground eigenvalue peak, although the original data employ exact values, not distributions. Although 
some of the large spread in the ground eigenvalue peak in natural porous materials might be contributed to by the inversion algorithm, we believe that this spread is likely to be due to the heterogeneity of materials and exchange between the bulk of the pore and finer surface features or clay agglomerates. Effective parameter-identification methods that simulate diffusion in pore space imaged by $\mathrm{x}$ ray microtomography may improve our understanding of the contrast in the spread of relaxation features in porous materials [62].

Although we report only the observation and use of nonground eigenvalues of relaxation in rocks, similar features are also observed in biological samples. For example, similar multimodal features are observable in 2D relaxation data of Warner [63], for lamb liver and kidney, and 1D relaxation data of Peled et al. [64], for the frog sciatic nerve. Such features can be extracted to convert relaxation times into pore size and surface sink strength that are physically more meaningful.

Future work will address the effect of internal magnetic field gradients on the observed eigenvalues, investigating the effects of diffusive coupling [36], investigation of more samples, faster spatially resolved confinement measurements, and confinement measurements at different degrees of fluid saturation in a variety of samples with this and related $T_{1}-T_{2}$ spatially resolved methods.

\section{CONCLUSIONS}

The fast-diffusion model is prevalent in the interpretation of magnetic resonance relaxation-time constants in porous materials. This conviction is challenged in this work. It is demonstrated that diffusion is not fast enough to completely remove the effects of nonground eigenvalues in relaxation measurements that begin with homogeneous magnetization. The relaxation-diffusion behavior in many practical samples is either close to, or in, the intermediatediffusion regime described by Brownstein and Tarr. It is therefore possible to observe and detect nonground eigenvalues of magnetic resonance relaxation in simple relaxation experiments commonly utilized in the laboratory and in the field. Nonground eigenvalues and their corresponding intensities may be employed in the calculation of pore size and surface relaxivities by matching numerical Brownstein-Tarr solutions with experimental relaxation data. Such a protocol may be utilized in any magnetic resonance measurements of porous materials that have at least one relaxation dimension. We present a phaseencoding imaging variant of this method that measures the imbibition confinement size in Berea sandstone.

The presence, detection, and utilization of nonground eigenvalues of relaxation in porous media can fundamentally change current practices in the measurement and analysis of porous-media relaxation data. For example, the relationship between relaxation-time constants and pore size may not be true in all samples. It is incomplete to analyze relaxation data as if only the ground eigenvalue is observable. These findings make it possible to reprocess a very large body of data on one- and multidimensional magnetic resonance relaxation of technological and biological samples. This work also encourages the development of magnetic resonance hardware, pulse sequences, and dataprocessing methods that can measure and detect such features.

\section{ACKNOWLEDGMENTS}

A.A. thanks the New Brunswick Innovation Fund for a Graduate Scholarship, the Association of Professional Engineers and Geoscientists of New Brunswick for an Advanced Studies Scholarship, the UNB School of Graduate Studies for a Gerald I. Goobie Chemical Engineering Scholarship, and the Danish Hydrocarbon Research and Technology Centre for funding. B.J.B. acknowledges the NSERC of Canada for a Discovery Grant and the Canada Chairs Program for a Chair in Material Science MRI. The authors thank Steve Cogswell from UNB Microscopy and Microanalysis for microscopy and Sarah Vashaee for discussions.

[1] G. R. Coates, L. Xiao, and M. G. Prammer, NMR Logging: Principles and Applications (Halliburton Energy Services, Houston, 1999).

[2] J. Mitchell, T. C. Chandrasekera, D. J. Holland, L. F. Gladden, and E. J. Fordham, Magnetic resonance imaging in laboratory petrophysical core analysis, Phys. Rep. 526, 165 (2013).

[3] A. Legchenko, Magnetic Resonance Imaging for Groundwater (John Wiley \& Sons, London, 2013).

[4] Y.-Q. Song, S. Ryu, and P. N. Sen, Determining multiple length scales in rocks, Nature 406, 178 (2000).

[5] R. Freedman and N. Heaton, Fluid characterization using nuclear magnetic resonance logging, Petrophysics 45, 241 (2004).

[6] W. S. Price, NMR Studies of Translational Motion (Cambridge University Press, Cambridge, 2009).

[7] B. A. Baldwin and R. L. King, in Spatially Resolved Magnetic Resonance (Wiley-VCH Verlag $\mathrm{GmbH}$, Weinheim, Germany, 1998), pp. 575-601.

[8] C. E. Muir and B. J. Balcom, in Annual Reports on NMR Spectroscopy (Academic Press, Burlington, 2012), pp. 81-113.

[9] M. Shakerian and B. J. Balcom, An MR/MRI compatible core holder with the RF probe immersed in the confining fluid, J. Magn. Reson. 286, 36 (2018).

[10] A. Afrough, M. Shakerian, M. S. Zamiri, B. MacMillan, F. Marica, B. Newling, L. Romero-Zerón, and B. J. Balcom, Magnetic-resonance imaging of high-pressure carbon dioxide displacement: Fluid/surface interaction and fluid behavior, SPE J. 23, 772 (2018). 
[11] R. L. Kleinberg, in Experimental Methods in the Physical Sciences (Academic Press, San Diego, 1999), pp. 337-385.

[12] J. R. Zimmerman and W. E. Brittin, Nuclear magnetic resonance studies in multiple phase systems: Lifetime of a water molecule in an adsorbing phase on silica gel, J. Phys. Chem. 61, 1328 (1957).

[13] L. Latour, R. Kleinberg, and A. Sezginer, Nuclear magnetic resonance properties of rocks at elevated temperatures, J. Colloid Interface Sci. 150, 535 (1992).

[14] W. E. Kenyon, P. I. Day, C. Straley, and J. F. Willemsen, A three-part study of NMR longitudinal relaxation properties of water-saturated sandstones, SPE Form. Eval. 3, 622 (1988).

[15] K. R. Brownstein and C. E. Tarr, Importance of classical diffusion in NMR studies of water in biological cells, Phys. Rev. A 19, 2446 (1979).

[16] Y.-Q. Song, Detection of the High Eigenmodes of Spin Diffusion in Porous Media, Phys. Rev. Lett. 85, 3878 (2000).

[17] N. V. Lisitza and Y.-Q. Song, The behavior of diffusion eigenmodes in the presence of internal magnetic field in porous media, J. Chem. Phys. 114, 9120 (2001).

[18] Y.-Q. Song, Pore sizes and pore connectivity in rocks using the effect of internal field, Magn. Reson. Imaging 19, 417 (2001).

[19] N. V. Lisitza and Y.-Q. Song, Manipulation of the diffusion eigenmodes in porous media, Phys. Rev. B 65, 172406 (2002).

[20] S. Davies and K. J. Packer, Pore-size distributions from nuclear magnetic resonance spin-lattice relaxation measurements of fluid-saturated porous solids. I. Theory and simulation, J. Appl. Phys. 67, 3163 (1990).

[21] S. Davies, M. Z. Kalam, K. J. Packer, and F. O. Zelaya, Pore-size distributions from nuclear magnetic resonance spin-lattice relaxation measurements of fluid-saturated porous solids. II. Applications to reservoir core samples, J. Appl. Phys. 67, 3171 (1990).

[22] K. Keating, A laboratory study to determine the effect of surface area and bead diameter on NMR relaxation rates of glass bead packs, Near Surf. Geophys. 12, 243 (2014).

[23] K. Dlubac, R. Knight, and K. Keating, A numerical study of the relationship between NMR relaxation and permeability in sands and gravels, Near Surf. Geophys. 12, 219 (2014).

[24] S. Falzone and K. Keating, A laboratory study to determine the effect of pore size, surface relaxivity, and saturation on $\mathrm{NMR} \mathrm{T}_{2}$ relaxation measurements, Near Surf. Geophys. 14, 57 (2016).

[25] M. Müller-Petke, R. Dlugosch, J. Lehmann-Horn, and M. Ronczka, Nuclear magnetic resonance average pore-size estimations outside the fast-diffusion regime, Geophysics 80, D195 (2015).

[26] R. Dlugosch, T. Günther, M. Müller-Petke, and U. Yaramanci, Improved prediction of hydraulic conductivity for coarse-grained, unconsolidated material from nuclear magnetic resonance, Geophysics 78, EN55 (2013).

[27] S. Costabel, C. Weidner, M. Müller-Petke, and G. Houben, Hydraulic characterisation of iron-oxide-coated sand and gravel based on nuclear magnetic resonance relaxation mode analyses, Hydrol. Earth Syst. Sci. 22, 1713 (2018).
[28] Z. Yu, Y. Zhang, L. Xiao, and G. Liao, Characterization of porous media by $T_{2}-T_{2}$ correlation beyond fast diffusion limit, Magn. Reson. Imaging 56, 19 (2019).

[29] M. D. Hürlimann, K. G. Helmer, L. L. Latour, and C. H. Sotak, Restricted diffusion in sedimentary rocks. Determination of surface-area-to-volume ratio and surface relaxivity, J. Magn. Reson. Ser. A 111, 169 (1994).

[30] A. Afrough, S. Vashaee, L. Romero Zerón, and B. Balcom, Absolute Measurement of Pore Size Based on Nonground Eigenstates in Magnetic-Resonance Relaxation, Phys. Rev. Appl. 11, 041002 (2019).

[31] H. C. Torrey, Bloch equations with diffusion terms, Phys. Rev. 104, 563 (1956).

[32] F. Bloch, Nuclear induction, Phys. Rev. 70, 460 (1946).

[33] J. Korringa, D. O. Seevers, and H. C. Torrey, Theory of spin pumping and relaxation in systems with a low concentration of electron spin resonance centers, Phys. Rev. 127, 1143 (1962).

[34] R. B. Bird, W. E. Stewart, and E. N. Lightfoot, Transport Phenomena, 2nd ed (John Wiley \& Sons, New York, 2002).

[35] See the Supplemental Material at http://link.aps.org/su pplemental/10.1103/PhysRevApplied.16.034040 for the effect of variation of the regularization parameter on $I\left(T_{1}, p, T_{1}, q\right)$ in Berea and Bentheimer sandstones, a simulated time-resolved magnetization profile in the intermediate diffusion regime, and the $T_{1}-T_{2}$ correlation map of Berea sandstone at $98.8 \mathrm{MHz}$.

[36] Y.-Q. Song, G. Carneiro, L. M. Schwartz, and D. L. Johnson, Experimental Identification of Diffusive Coupling Using 2D NMR, Phys. Rev. Lett. 113, 235503 (2014).

[37] A. D. Bain, Chemical exchange in NMR, Prog. Nucl. Magn. Reson. Spectrosc. 43, 63 (2003).

[38] J. H. Lee, C. Labadie, C. S. Springer, and G. S. Harbison, Two-dimensional inverse laplace transform NMR: Altered relaxation times allow detection of exchange correlation, J. Am. Chem. Soc. 115, 7761 (1993).

[39] Y.-Q. Song, L. Venkataramanan, M. D. Hürlimann, M. Flaum, P. Frulla, and C. Straley, $\mathrm{T}_{1}-\mathrm{T}_{2}$ correlation spectra obtained using a fast Two-dimensional laplace inversion, J. Magn. Reson. 154, 261 (2002).

[40] K. M. Song, J. Mitchell, H. Jaffel, and L. F. Gladden, Simultaneous monitoring of hydration kinetics, microstructural evolution, and surface interactions in hydrating gypsum plaster in the presence of additives, J. Mater. Sci. 45, 5282 (2010).

[41] R. Enjilela, J. Guo, B. MacMillan, F. Marica, A. Afrough, and $\mathrm{B}$. Balcom, $\mathrm{T}_{1}-\mathrm{T}_{2}{ }^{*}$ relaxation correlation measurements, J. Magn. Reson. 326, 106961 (2021).

[42] A. A. Istratov and O. F. Vyvenko, Exponential analysis in physical phenomena, Rev. Sci. Instrum. 70, 1233 (1999).

[43] J. Mitchell, T. C. Chandrasekera, and L. F. Gladden, Numerical estimation of relaxation and diffusion distributions in two dimensions, Prog. Nucl. Magn. Reson. Spectrosc. 62, 34 (2012).

[44] J. Mitchell, L. F. Gladden, T. C. Chandrasekera, and E. J. Fordham, Low-field permanent magnets for industrial process and quality control, Prog. Nucl. Magn. Reson. Spectrosc. 76, 1 (2014).

[45] L. Venkataramanan, Yi-Qiao Song, and M. D. Hurlimann, Solving fredholm integrals of the first kind with tensor 
product structure in 2 and 2.5 dimensions, IEEE Trans. Signal Process. 50, 1017 (2002).

[46] P. C. Hansen, Discrete Inverse Problems: Insight and Algorithms (Society for Industrial and Applied Mathematics, Philadelphia, 2010).

[47] C. R. Vogel, Computational Methods for Inverse Problems (Society for Industrial and Applied Mathematics, Philadelphia, 2002).

[48] F. Marica, A. Afrough, D. Green, L. Romero-Zerón, and B. J. Balcom, Direct Magnetic Resonance Measurement of Average Pore Size (Society of Core Analysts, Pau, France, 2019).

[49] O. V. Petrov, G. Ersland, and B. J. Balcom, T 2 distribution mapping profiles with phase-encode MRI, J. Magn. Reson. 209, 39 (2011).

[50] M. H. Levitt, Composite pulses, Prog. Nucl. Magn. Reson. Spectrosc. 18, 61 (1986).

[51] M. H. Levitt, in Encyclopedia of Nuclear Magnetic Resonance, edited by D. M. Grant, R. K. Harris (Wiley, Chichester, 1996), Vol. 2, pp.1396-1411.

[52] K. Livo, M. Prasad, and T. R. Graham, Quantification of dissolved $\mathrm{O}_{2}$ in bulk aqueous solutions and porous media using NMR relaxometry, Sci. Rep. 11, 290 (2021).

[53] M. Holz, S. R. Heil, and A. Sacco, Temperature-dependent self-diffusion coefficients of water and six selected molecular liquids for calibration in accurate ${ }^{1} \mathrm{H}$ NMR PFG measurements, Phys. Chem. Chem. Phys. 2, 4740 (2000).

[54] H. Safari, B. Balcom, and A. Afrough, Characterization of Pore and Grain Size Distributions in Porous Geological Samples (University of New Brunswick, Fredericton, 2021). https://doi.org/10.25545/A19OPN

[55] H. Safari, B. J. Balcom, and A. Afrough, Characterization of pore and grain size distributions in porous geological samples - An image processing workflow, Comput. Geosci. 156, 104895 (2021).

[56] G. C. Borgia, R. J. S. Brown, and P. Fantazzini, Uniformpenalty inversion of multiexponential decay data, J. Magn. Reson. 132, 65 (1998).

[57] J. Mitchell, T. C. Chandrasekera, M. L. Johns, L. F. Gladden, and E. J. Fordham, Nuclear magnetic resonance relaxation and diffusion in the presence of internal gradients: The effect of magnetic field strength, Phys. Rev. E 81, 026101 (2010).

[58] T. G. Kolda, R. M. Lewis, and V. Torczon, Optimization by direct search: New perspectives on some classical and modern methods, SIAM Rev. 45, 385 (2003).

[59] T. M. Geffen, D. R. Parrish, G. W. Haynes, and R. A. Morse, Efficiency of gas displacement from porous media by liquid flooding, J. Pet. Technol. 4, 29 (1952).

[60] F. A. L. Dullien, Porous Media: Fluid Transport and Pore Structure (Academic Press, San Diego, 1992).

[61] J. E. Maneval, M. L. Nelson, L. W. Thrane, S. L. Codd, and J. D. Seymour, A two-region transport model for interpreting $\mathrm{T}_{1}-\mathrm{T}_{2}$ measurements in complex systems, J. Magn. Reson. 308, 106592 (2019).

[62] R. Li, I. Shikhov, and C. Arns, Solving Multiphysics, Multiparameter, Multimodal Inverse Problems: An Application to NMR Relaxation in Porous Media, Phys. Rev. Appl. 15, 054003 (2021).

[63] J. Warner, Developments in 2D NMR Relaxometry and its Application to Biological Tissue (University of East Anglia, Norwich, 2010).

[64] S. Peled, D. G. Cory, S. A. Raymond, D. A. Kirschner, and F. A. Jolesz, Water diffusion, $\mathrm{T}_{2}$, and compartmentation in frog sciatic nerve, Magn. Reson. Med. 42, 911 (1999). 